\title{
Bivariant cycle cohomology
}

\author{
E.M. Friedlander ${ }^{1}$ and V.Voevodsky
}

\section{Contents}

1 Introduction. . . . . . . . . . . . . . . . 1

2 Presheaves of relative cycles. ................ 3

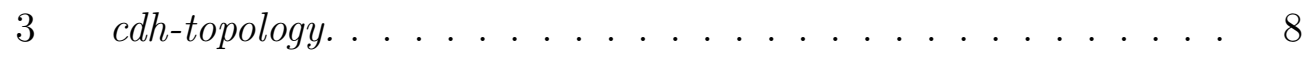

4 Bivariant cycle cohomology. ............. . 13

5 Pretheories. ...................... 19

6 Moving Lemma. . . . . . . . . . . . . . . . . . . . . . . . 29

$7 \quad$ Duality. . . . . . . . . . . . . . . . . 37

8 Properties and pairings. . . . . . . . . . . . . . . 41

9 Motivic cohomology and homology. . . . . . . . . . . . 47

\section{Introduction.}

The precursor of our bivariant cycle cohomology theory is the graded Chow group $A_{*}(X)$ of rational equivalence classes of algebraic cycles on a scheme $X$ over a field $k$. In [1], S. Bloch introduced the higher Chow groups $C H^{*}(X, *)$ in order to extend to higher algebraic K-theory the relation established by A. Grothendieck between $K_{0}(X)$ and $A_{*}(X)$. More recently, Lawson homology theory for complex algebraic varieties has been developed (cf. [13], [4]), in which the role of rational equivalence is replaced by algebraic equivalence, and a bivariant extension $L^{*} H^{*}(Y, X)$ [8] has been introduced. This more topological approach suggested a duality relating covariant and contravariant theories as established in [7].

Our bivariant cycle cohomology groups $A_{r, i}(Y, X)$, defined for schemes $Y, X$ of finite type over a field $k$, satisfy $\left.A_{r, i}(\operatorname{Spec}(k), X)\right)=C H^{n-r}(X, i)$ whenever $X$ is an affine scheme over $k$ of pure dimension $n$. This bivariant theory is a somewhat more sophisticated version of a theory briefly introduced by the first author and O. Gabber in [6]. The added sophistication enables us to prove numerous good properties of this theory, most notably

\footnotetext{
${ }^{1}$ Partially supported by the N.S.F. and NSA Grant \#MDA904-
} 
localization (Theorem 5.11) and duality (Theorem 8.2), which suggest that $A_{*, *}(Y, X)$ should be useful in various contexts. For example, we envision that the bivariance and duality of $A_{*, *}(Y, X)$ will be reflected in a close relationship to the algebraic K-theories of coherent sheaves and of locally free sheaves on schemes of finite type over a field $k$. Moreover, we establish pairings for our theory whose analogues in Lawson homology have proved useful in studying algebraic cycles (cf. [10],[3]).

The final section presents a formulation of motivic cohomology and homology in terms of $A_{*, *}(Y, X)$.

The development of our theory requires machinery developed recently by the second author, partially in joint work with A. Suslin. As we incorporate the functoriality of cycles [21], pretheories [23], sheaves for the cdh-topology [21], and homology vanishing theorems [23], we endeavor to prodvide an introduction to these ideas by summarizing relevant results from these papers and by providing complete proofs of modified results which we require.

A quick overview of this paper can be obtained from a glance at the table of contents. To conclude this introduction, we give a somewhat more detailed summary of the contents of the various sections of our paper. Section 2 recalls from [21] the functoriality of the presheaf $z_{\text {equi }}(X, r)$ which sends a smooth scheme $U$ of finite type over $k$ to the group of cycles on $X \times$ $U$ equidimensional of relative dimension $r$ over $k$. For $X$ projective, this is closely related to the functor represented by the Chow monoid $\mathcal{C}_{r}(X)$ of effective $r$-cycles on $X$. Despite the proliferation of notation, we find it very convenient to consider the related presheaf $z_{\text {equi }}(Y, X, r)$ defined by $z_{\text {equi }}(Y, X, r)(U)=z_{\text {equi }}(X, r)(Y \times U)$.

Following [22], we consider for a presheaf $F$ the complex of presheaves $\underline{C}_{*}(F)$, sending a scheme $U$ of finite type over $k$ to $F\left(U \times \Delta^{\bullet}\right)$, and the associated homology presheaves $\underline{h}_{i}(F)$. In particular, the "naive" bivariant theory $\underline{h}_{i}\left(z_{\text {equi }}(X, r)(Y)\right.$ (closely related to a construction of [6]) is modified to satisfy "cdh-descent", resulting in the definition (Definition 3.9)

$$
A_{r, i}(Y, X)=\mathbf{H}^{-i}\left(Y, \underline{C}_{*}\left(z_{\text {equi }}(X, r)_{c d h}\right) .\right.
$$

The role of the cdh-topology, more flexible than the Zariski topology, is to permit as coverings surjective maps which arise in resolving the singularities of $Y$.

As developed by the second author in [23], a pretheory is a presheaf $F$ privided with well-behaved transfer maps. Such a structure has the following 
remarkable property (cf. Theorem 5.5): if the associated cdh-sheaf $F_{c d h}$ vanishes, then $\underline{C}_{*}(F)_{Z a r}=0$. This result requires that " $k$ admit resolution of singularities" ( a precise definition of what it means is given in Definition 3.4). The importance of this property is seen by the ease with which the difficult property of localization for $A_{*, *}(Y, X)$ with respect to the second argument follows: one applies this vanishing result to the pretheory $\operatorname{coker}\left(i^{*}\right.$ : $\left.z_{\text {equi }}(X, r) \rightarrow z_{\text {equi }}(X-W, r)\right)$ associated to a closed subscheme $W \subset X$ of $X$ over such a field $k$.

In Section 6, we recast in functorial language the recent "moving lemma for families" established by the first author and H.B. Lawson in [9], leading to the duality theorems of Section 7. For projective, smooth schemes $Y, X$ over an arbitrary field $k$, Theorem 7.1 provides isomorphisms of presheaves $\underline{h}_{i}\left(z_{\text {equi }}(Y, X, r)\right) \simeq \underline{h}_{i}\left(z_{\text {equi }}(X \times Y, r+n)\right)$. Theorem 8.2 asserts that if $k$ admits resolution of singularities, then there are canonical isomorphisms

$$
\left.A_{r, i}(Y \times U, X) \rightarrow A_{r+n, i}(X \times U)\right)
$$

for any smooth scheme $U$ of pure dimension $n$ over $k$. Other desired properties established in Section 8 include homotopy invariance, suspension and cosuspension isomorphisms, and a Gysin exact triangle. We also provide three pairings for our theory: the first is the direct analogue of the pairing that gives operations in Lawson homology [11], the second is a multiplicative pairing inspired by the multiplicative structure in morphic cohomology [8], and the final one is a composition product viewed in the context of Lawson homology as composition of correspondences.

Because of its good properties and its relationship to higher algebraic $K$ theory, one frequently views the higher Chow groups $C H^{n-r}(X, i)$ as at least one version of "motivic cohomology". Indeed, these higher Chow groups are a theory of "homology with locally compact supports." In the final section, we formulate four theories - cohomology/homology with/without compact supports - in terms of our bivariant cycle cohomology.

\section{Presheaves of relative cycles.}

Let $k$ be a field. For a scheme of finite type $Y$ over $k$ we denote by $C y c l(Y)$ the group of cycles on $Y$ (i.e. the free abelian group generated by closed integral subschemes of $Y$ ). For a closed subscheme $Z$ of $Y$ we define a cycle 
$\operatorname{cycl}_{Y}(Z)$ of $Z$ in $Y$ as the formal linear combination $\sum m_{i} Z_{i}$ where $Z_{i}$ are (reduced) irreducible components of $Z$ and $m_{i}$ is the length of the local ring $\mathcal{O}_{Z, Z_{i}}$ (note that in our case it is just the dimension of the finite algebra $\mathcal{O}(Z) \otimes_{\mathcal{O}\left(Z_{i}\right)} F\left(Z_{i}\right)$ over the field $F\left(Z_{i}\right)$ of functions on $\left.Z_{i}\right)$.

For a smooth scheme $U$ over $k$, a scheme of finite type $X$ over $k$ and an integer $r \geq 0$ we denote by $z_{\text {equi }}(X, r)(U)$ the subgroup in the group of cycles $\operatorname{Cycl}(X \times U)$ generated by closed integral subschemes $Z$ of $X \times U$ which are equidimensional of relative dimension $r$ over $U$ (note that it means in particular that $Z$ dominates a connected component of $U$ ). We denote further by $z_{\text {equi }}^{\text {eff }}(X, r)(U)$ the submonoid of effective cycles in $z_{\text {equi }}(X, r)(U)$.

The groups $z_{\text {equi }}(X, r)(U), z_{\text {equi }}^{\text {eff }}(X, r)(U)$ were considered in more general context of schemes of finite type over a Noetherian scheme in [21]. We will recall now some of the results obtained there.

For any morphism $f: U^{\prime} \rightarrow U$ there is a homomorphism cycl $(f)$ : $z_{\text {equi }}(X, r)(U) \rightarrow z_{\text {equi }}(X, r)\left(U^{\prime}\right)$ such that the following conditions hold:

1. For a composable pair of morphisms $U^{\prime \prime} \stackrel{g}{\rightarrow} U^{\prime} \stackrel{f}{\rightarrow} U$ one has $\operatorname{cycl}(f g)=$ $\operatorname{cycl}(g) \operatorname{cycl}(f)$.

2. For a dominant morphism $f: U^{\prime} \rightarrow U$ one has

$$
\operatorname{cycl}(f)\left(\sum n_{i} Z_{i}\right)=\sum n_{i} c y c l_{X \times U^{\prime}}\left(Z_{i} \times_{U} U^{\prime}\right)
$$

where $\operatorname{cycl}_{X \times U^{\prime}}\left(Z_{i} \times_{U} U^{\prime}\right)$ is the cycle associated with the closed subscheme $Z_{i} \times_{U} U^{\prime}$ in $X \times U^{\prime}$.

3. For any morphism $f: U^{\prime} \rightarrow U$ and a closed susbscheme $Z$ of $X \times U$ which is flat and equidimensional of relative dimension $r$ over $U$ one has

$$
\operatorname{cycl}(f)\left(\operatorname{cycl}_{X \times U}(Z)\right)=\operatorname{cycl}_{X \times U^{\prime}}\left(Z \times_{U} U^{\prime}\right) .
$$

One can verify easily that homomorphisms $\operatorname{cycl}(f)$ are uniquely determined by the conditions (1)-(3) above. Note that the condition (1) implies that we may consider $z_{\text {equi }}(X, r)$ as a presheaf of abelian groups on the category $S m / k$ of smooth schemes over $k$.

Since for an effective cycle $\mathcal{Z}$ the cycle $\operatorname{cycl}(f)(\mathcal{Z})$ is effective the submonoids $z_{\text {equi }}^{\text {eff }}(X, r)(U)$ of effective cycles in $z_{\text {equi }}(X, r)(U)$ form a subpresheaf $z_{\text {equi }}^{\text {eff }}(X, r)$ of $z_{\text {equi }}(X, r)(U)$. 
Proposition 2.1 For a cycle $\mathcal{Z}$ in $z_{\text {equi }}(X, r)(U)$, let $p_{*}(\mathcal{Z})$ denote the pushforward of $\mathcal{Z}$ to a cycle on $Y \times U$ for a proper morphism $p: X \rightarrow Y$; let $q^{*}(\mathcal{Z})$ denote the flat pull-back of $\mathcal{Z}$ to a cycle on $W \times U$ for a flat equidimensional morphism $q: W \rightarrow X$ of relative dimension $n$ of schemes of finite type over $k$. Then

$$
p_{*}(\mathcal{Z}) \in z_{\text {equi }}(Y, r)(U) \quad, \quad q^{*}(\mathcal{Z}) \in z_{\text {equi }}(W, r+n)(U) .
$$

Moreover, for any morphism of smooth schemes $f: U^{\prime} \rightarrow U$ one has

$$
\operatorname{cycl}(f)\left(p_{*}(\mathcal{Z})\right)=p_{*}(\operatorname{cycl}(f)(\mathcal{Z})), \operatorname{cycl}(f)\left(q^{*}(\mathcal{Z})\right)=q^{*}(\operatorname{cycl}(f)(\mathcal{Z})) .
$$

Proof: The assertions for proper push-forward are a special case of [21, Prop. 3.6.2(2)], whereas that for flat pull-back follow from [21, Lemma 3.6.4].

Proposition 2.1 implies that for any proper morphism $p: X \rightarrow Y$ of schemes of finite type over $k$ there is a homomorphism of presheaves $p$ : $z_{\text {equi }}(X, r) \rightarrow z_{\text {equi }}(Y, r)$. Clearly for a composable pair of morphisms $X \stackrel{p_{1}}{\rightarrow}$ $Y \stackrel{p_{2}}{\rightarrow} Z$ one has $\left(p_{2} p_{1}\right)_{*}=p_{2 *} p_{1 *}$. Similarly, for a flat equidimensional morphism $q: W \rightarrow X, q^{*}$ is a morphism of presheaves $z_{\text {equi }}(Y, r) \rightarrow z_{\text {equi }}(X, r+n)$ and for a composable pair of morphisms $X \stackrel{q_{1}}{\rightarrow} Y \stackrel{q_{2}}{\rightarrow} Z$ we have $\left(q_{2} q_{1}\right)^{*}=q_{1}^{*} q_{2}^{*}$.

Let $X$ be a scheme of finite type over $k, U$ be a smooth equidimensional scheme of finite type over $k$ and $V$ be a smooth scheme over $k$. Let $\mathcal{Z}$ be an element of $z_{\text {equi }}(X, r)(U \times V)$. Considered as a cycle on $X \times U \times V$ it clearly belongs to $z_{\text {equi }}(X \times U, r+\operatorname{dim}(U))(V)$. By [21, Th. 3.7.3], we have for any morphism $f: V^{\prime} \rightarrow V$ of smooth schemes over $k$ the following equality of cycles on $X \times U \times V^{\prime}$ :

$$
\operatorname{cycl}(f)(\mathcal{Z})=\operatorname{cycl}\left(I d_{U} \times f\right)(\mathcal{Z}) .
$$

For any $X, U, V$, denote by $z_{\text {equi }}(U, X, r)(V)$ the group $z_{\text {equi }}(X, r)(U \times V)$. Then $z_{\text {equi }}(U, X, r)$ is a presheaf on $S m / k$ which is contravariantly functorial with respect to $U$. The above equality asserts that we have a canonical embedding of presheaves

$$
\mathcal{D}: z_{\text {equi }}(U, X, r) \rightarrow z_{\text {equi }}(X \times U, r+\operatorname{dim}(U)) .
$$

One can verify easily that homomorphisms $\mathcal{D}$ are consistent with covariant (resp. contravariant) functoriality of both presheaves with respect to proper (resp. flat equidimensional) morphisms $X \rightarrow X^{\prime}$. 
Consider now the case of a projective scheme $X \subset \mathbf{P}^{m}$ over $k$. For an effective cycle $\mathcal{Z}=\sum n_{i} Z_{i} \in z_{\text {equi }}^{\text {eff }}(X, r)(\operatorname{Spec}(k))$ on $X$ we define its degree $\operatorname{deg}(\mathcal{Z})=\operatorname{deg}_{i_{X}}(\mathcal{Z})$ as the $\operatorname{sum} \sum n_{i} \operatorname{deg}_{i_{X}}\left(Z_{i}\right)$ where $\operatorname{deg}_{i_{X}}\left(Z_{i}\right)$ is the degree of the reduced closed subscheme $i_{X}\left(Z_{i}\right)$ in $\mathbf{P}_{k}^{m}$. The lemma below follows easily from the invariance of degree in flat families.

Lemma 2.2 Let $U$ be a smooth connected scheme over $k$ and $i_{X}: X \rightarrow \mathbf{P}^{m}$ be a projective scheme over $k$. Let further $\mathcal{Z}$ be an element of $z_{\text {equi }}(X, r)(U)$ and

$$
\begin{aligned}
& u_{1}: \operatorname{Spec}\left(k_{1}\right) \rightarrow U \\
& u_{2}: \operatorname{Spec}\left(k_{2}\right) \rightarrow U
\end{aligned}
$$

be two points of $U$. Then one has

$$
\operatorname{deg}\left(\operatorname{cycl}\left(u_{1}\right)(\mathcal{Z})\right)=\operatorname{deg}\left(\operatorname{cycl}\left(u_{2}\right)(\mathcal{Z})\right) .
$$

where the degree on the right hand side (resp. left hand side) is considered with respect to the closed embedding $i_{X} \times_{\operatorname{Spec}(k)} \operatorname{Spec}\left(k_{2}\right) \quad\left(\operatorname{resp} . i_{X} \times_{\operatorname{Spec}(k)}\right.$ $\left.\operatorname{Spec}\left(k_{1}\right)\right)$.

In the situation of Lemma 2.2 we will use the notation $\operatorname{deg}(\mathcal{Z})$ for the degree of the fibers of $\mathcal{Z}$. Denote by $z_{\text {equi }}^{\text {eff }}(X, r, d)\left(\operatorname{resp} . z_{\text {equi }}^{\text {eff }}(X, r, \leq d)(U)\right)$ the subset in $z_{\text {equi }}^{e f f}(X, r)(U)$ which consists of cycles of degree $d$ (resp. $\leq$ $d)$. By Lemma $2.2 z_{\text {equi }}^{\text {eff }}(X, r, \leq d)$ and $z_{\text {equi }}^{\text {eff }}(X, r, d)$ are subpresheaves in $z_{\text {equi }}^{\text {eff }}(X, d)$.

We may now relate our presheaves $z_{\text {equi }}^{\text {eff }}(X, r)$ to the presheaves represented by Chow varieties. Let us recall briefly the construction of the Chow variety of effective cycles of dimension $r$ and degree $d$ on a projective scheme $X \subset \mathbf{P}^{m}$ over a field $k$.

Let $\left(\mathbf{P}^{m}\right)^{r+1}$ be the be the product of projective spaces which we consider as the space which parametrizes families $\left(L_{0}, \ldots, L_{r}\right)$ of $r+1$ hyperplanes in $\mathbf{P}^{m}$. Consider the incidence correspondence

$$
\Gamma \subset \mathbf{P}^{m} \times\left(\mathbf{P}^{m}\right)^{r+1}
$$

which consists of pairs of the form $\left(x,\left(L_{0}, \ldots, L_{r}\right)\right)$ such that $x \in L_{0} \cap \ldots \cap L_{r}$. One can easily see that the projection $p r_{1}: \Gamma \rightarrow \mathbf{P}^{m}$ is smooth. Therefore for any cycle $\mathcal{Z}$ of dimension $r$ on $\mathbf{P}^{m}$ there is defined a cycle $\operatorname{Chow}(\mathcal{Z})=$ 
$\left(p r_{2}\right)_{*}\left(p r_{1}\right)^{*}(\mathcal{Z})$ on $\left(\mathbf{P}^{m}\right)^{r+1}$. One can verify easily that $\operatorname{Chow}(\mathcal{Z})$ is a cycle of codimension 1 and if $\operatorname{deg}(\mathcal{Z})=d$ then $\operatorname{Chow}(\mathcal{Z})$ is a cycle of degree $(d, \ldots, d)$. Since effective cycles of codimension 1 and given degree on a product of projective spaces are parametrized by a projective space, this gives us a map

$$
z_{\text {equi }}^{e f f}\left(\mathbf{P}^{m}, r, d\right)(\operatorname{Spec}(\bar{k})) \rightarrow \mathbf{P}^{N(m, r, d)}(\bar{k}) .
$$

It is well known in the classical theory of algebraic cycles (see [19] for a very classical approach or [21] for a very modern one) that this map is injective and its image coincides with the set of $\bar{k}$-points of a closed (reduced) subscheme $C_{r, d}\left(\mathbf{P}^{m}\right)$ in $\mathbf{P}^{N(m, r, d)}$. Moreover for any closed subscheme $X$ in $\mathbf{P}^{m}$ the set of $\bar{k}$-points of $C_{r, d}\left(\mathbf{P}^{m}\right)$ which correspond to cycles with support on $X$ coincides with the set of $\bar{k}$-points of a closed (reduced) subscheme $C_{r, d}(X)$ in $C_{r, d}\left(\mathbf{P}^{m}\right)$ which is called the Chow variety of cycles of degree $d$ and dimesnion $r$ on $X$.

Since the construction of the Chow map above only uses the operations of flat pull-back and proper push-forward on cycles, Proposition 2.1 implies that the obvious relative version of this map is well defined and gives a homomorphism of presheaves

$$
z_{\text {equi }}^{e f f}\left(\mathbf{P}^{m}, r, d\right) \rightarrow z_{\text {equi }}^{e f f}\left(\left(\mathbf{P}^{m}\right)^{r+1}, m r+m-1,(d, \ldots, d)\right) .
$$

The right hand side presheaf is representable on the category $S m / k$ by the projective space $\mathbf{P}^{N(m, r, d)}$ and we conclude that for any smooth scheme $U$ over $k$ there is a map

$$
z_{\text {equi }}^{e f f}(X, r, d)(U) \rightarrow \operatorname{Hom}\left(U, C_{r, d}(X)\right)
$$

and that these maps give us a monomorphism from the presheaf $z_{\text {equi }}^{e f f}(X, r, d)$ to the presheaf represented by $C_{r, d}(X)$ (this construction was explored in [4] and considered in the more general context of normal schemes in [8]).

We let $\mathcal{C}_{r}(X)$ denote the Chow monoid

$$
\mathcal{C}_{r}(X) \equiv \coprod_{d \geq 0} C_{r, d}(X)
$$

of effective $r$-cycles on $X$. Up to weak normalization, $\mathcal{C}_{r}(X)$ does not depend upon the projective embedding $X \subset \mathbf{P}^{m}$. 
Proposition 2.3 Let $X$ be a projective scheme and $U$ a quasi-projective scheme over $k$. Then every $\mathcal{Z} \in z_{\text {equi }}(X, r)^{\text {eff }}(U)$ determines a morphism $f_{\mathcal{Z}}: U \rightarrow \mathcal{C}_{r}(X)$. If $f: U \rightarrow \mathcal{C}_{r}(X)$ is a morphism and if $u \in U$, then the effective cycle $\mathcal{Z}_{f(u)}$ with Chow point $f(u)$ is defined over a field $k\left(\mathcal{Z}_{f(u)}\right)$ which is a finite radiciel extension of the residue field $k(f(u))$. Such a morphism $f: U \rightarrow \mathcal{C}_{r}(X)$ determines $\mathcal{Z}_{f} \in z_{\text {equi }}(X, r)^{\text {eff }}(U)$ if and only if $k\left(\mathcal{Z}_{f(\eta)}\right)=k(f(\eta))$ for every generic point $\eta \in U$. Finally, for any $\mathcal{Z} \in z_{\text {equi }}(X, r)^{e f f}(U)$,

$$
\mathcal{Z}=\mathcal{Z}_{f_{\mathcal{Z}}}, \quad f=f_{\mathcal{Z}_{f}} .
$$

Proof: This is proved in $[4,1.4]$

In particular, if $k$ is a field of characteristic zero, then Proposition 2.3 implies that effective cycles on $U \times X$ equidimensional over the smooth scheme $U$ of relative diemsion $r$ correspond exactly to maps from $U$ to $\mathcal{C}_{r}(X)$. The following example shows that in arbitrary charcteristic this is no longer true.

Example 2.4 (cf. [19]) Let $k=F\left(t_{1}, t_{2}\right)$ where $F$ is an algebraically closed field of characteristic $p>0$. Consider the 0 -cycle $\mathcal{Z}$ on $\mathbf{P}_{F}^{2}$ which corresponds to the closed reduced subscheme given by the equations

$$
\begin{aligned}
& x^{p}=t_{1} z^{p} \\
& y^{p}=t_{2} z^{p}
\end{aligned}
$$

where $x, y, z$ are homogeneous coordinates on $\mathbf{P}^{2}$. One can verify easily that Chow $(\mathcal{Z})$ is the cycle in $\mathbf{P}_{F}^{2}$ (with homogeneous coordinates $a, b, c$ ) of the form $p D$ where $D$ is the cycle of the closed subscheme given by the equation $t_{1} a^{p}+t_{2} b^{p}+c^{p}=0$. It follows immediately from definition of Chow varieties that $D$ belongs to $C_{0, p}\left(\mathbf{P}_{F}^{2}\right)$. On the other hand there is no cycle $\mathcal{W}$ on $\mathbf{P}_{F}^{2}$ such that $p \mathcal{W}=\mathcal{Z}$.

Remark 2.5 Another construction of a presheaf of r-cycles on $X$ for a quasiprojective scheme $X$ was given in [6]. We will discuss its relations to our definition at the end of the next section.

\section{3 cdh-topology.}

In this section we remind the definition of cdh-topology given in [21]. In order to work with possibly singular schemes, we use maps of the following form which arise in resoultion of singularities 
Definition 3.1 Let $X$ be a scheme of finite type over $k$ and $Z \subset X$ be a closed subscheme in $X$ which does not contain generic points of irreducible components of $X$. An abstract blow-up of $X$ with center in $Z$ is a proper surjective morphism $p: X^{\prime} \rightarrow X$ such that $p^{-1}(X-Z)_{\text {red }} \rightarrow(X-Z)_{\text {red }}$ is an isomorphism.

Denote by $S c h / k$ the category of schemes of finite type over $k$. We recall the Nisnevich topology introduced by Y. Nisnevich in [16] and its modification the $c d h$-topology (the completely decomposed h-topology) introduced in [21].

Definition 3.2 The Nisnevich topology is the minimal Grothendieck topology on Sch/k such that the following type of covering is a Nisnevich covering: etale coverings $\left\{U_{i} \stackrel{p_{i}}{\rightarrow} X\right\}$ such that for any point $x$ of $X$ there is a point $x_{i}$ on one of the $U_{i}$ such that $p_{i}\left(x_{i}\right)=x$ and the morphism $\operatorname{Spec}\left(k\left(x_{i}\right)\right) \rightarrow \operatorname{Spec}(k(x))$ is an isomorphism.

The cdh-topology on $S c h / k$ is the minimal Grothendieck topology on this category such that Nisnevich coverings and the following type of covering are cdh-covering: coverings of the form

$$
Y \coprod X_{1} \stackrel{p_{Y}}{\rightrightarrows i_{X_{1}}} X
$$

such that $p_{Y}$ is a proper morphism, $i_{X_{1}}$ is a closed embedding and the morphism $p_{Y}^{-1}\left(X-X_{1}\right) \rightarrow X-X_{1}$ is an isomorphism.

Thus, the cdh-topology permits abstract blow-ups as coverings as well as the disjoint union of the embeddings of irreducible components of a reducible scheme.

Note in particular, that for any scheme $X$ the closed embedding $X_{\text {red }} \rightarrow X$ where $X_{\text {red }}$ is a maximal reduced subscheme of $X$ is a cdh-covering. Thus, working with the cdh-topology, we do not see any difference between $X$ and $X_{\text {red }}$. We will often use this fact below without additional references.

The following elementary result about the cdh-topology will be used in the proof of Theorem 5.5 (which uses Theorem 5.1 and an investigation of the relationship of the cohomological behaviour of sheaves on the Nisnevich topology and sheaves on the cdh-topology).

Lemma 3.3 Let $F$ be a sheaf in Nisnevich topology on Sch $/ k$ such that $F_{c d h}=0, X$ be a scheme of finite type over $k$ and $U \rightarrow X$ be a Nisnevich 
covering of $X$. Then for any element $a \in F(U)$, there is an abstract blowup $X^{\prime} \rightarrow X$ with center in $Z \subset X$ such that $\operatorname{dim}(Z)<\operatorname{dim}(X)$ and the restriction of a to $U \times_{X} X^{\prime}$ equals zero.

Proof: Using the definition of the cdh-topology and the fact that $F$ is assumed to be a Nisnevich sheaf, we conclude for any $a \in F(U)$ that there is some abstract blow-up $p: U^{\prime} \rightarrow U$ with $p^{*}(a)=0$. We apply the platification theorem [17] to obtain an abstract blow-up $X^{\prime} \rightarrow X$ with center in a closed subscheme $Z \subset X$ which does not contain generic points of $X$ such that the proper transform $\tilde{U}^{\prime} \rightarrow X^{\prime}$ of $U^{\prime} \rightarrow X$ with respect to this blow-up is flat over $X^{\prime}$. One can easily see that we have $\tilde{U}^{\prime}=U \times_{X} X^{\prime}$, so that $U \times_{X} X^{\prime}$ factors through $U^{\prime}$.

To prove more about cdh-topology we will often have to assume that the base field $k$ "admits resolution of singularities". More precisely one has.

Definition 3.4 Let $k$ be a field. We say that $k$ admits resolution of singularities if the following two conditions hold:

1. For any scheme of finite type $X$ over $k$ there is a proper surjective morphism $Y \rightarrow X$ such that $U$ is a smooth scheme over $k$.

2. For any smooth scheme $X$ over $k$ and an abstract blow-up $q: X^{\prime} \rightarrow X$ there exists a sequence of blow-up $p: X_{n} \rightarrow \ldots \rightarrow X_{1}=X$ with smooth centers such that $p$ factors through $q$

The following proposition follows immediately from standard results on resolution of singularities in characteristic zero ([12]).

Proposition 3.5 Any field of characteristic zero admits resolution of singularities in the sense of Definition 3.4.

Note that any field which admits resolution of singularities in the sense of Definition 3.4 is a perfect field.

Let $\pi:(S c h / k)_{c d h} \rightarrow(S m / k)_{Z a r}$ be the natural morphism of sites. Let further

$$
\pi^{*}: S h v\left((S m / k)_{Z a r}\right) \rightarrow \operatorname{Shv}\left((S c h / k)_{c d h}\right)
$$

be the corresponding functor of inverse image on the categories of sheaves. We will use the following simple lemma. 
Lemma 3.6 Let $k$ be a field which admits resolution of singularities. Then the functor of inverse image

$$
\pi^{*}: \operatorname{Shv}\left((S m / k)_{Z a r}\right) \rightarrow \operatorname{Shv}\left((S c h / k)_{c d h}\right)
$$

is exact.

Proof: Consider the minimal Grothendieck topology $t$ on the category $S m / k$ such that all cdh-coverings of the form $U^{\prime} \rightarrow U$ with both $U^{\prime}$ and $U$ being smooth are $t$-coverings. Clearly we have a morphism of sites

$$
\pi_{0}:(S c h / k)_{c d h} \rightarrow(S m / k)_{t}
$$

and a decomposition of $\pi^{*}$ of the form:

$$
S h v\left((S m / k)_{Z a r}\right) \rightarrow S h v\left((S m / k)_{t}\right) \stackrel{\pi_{0}^{*}}{\rightarrow} S h v\left((S c h / k)_{c d h}\right)
$$

where the first arrow is the functor of associated $t$-sheaf. Note that this functor is exact. Resolution of singularities implies easily that the functor $\pi_{0}^{*}$ is an equivalence. Thus $\pi^{*}$ is exact.

Remark 3.7 The statement of Lemma 3.6 would be false if one considers the Zariski topology on $S c h / k$ instead of cdh-topology. The problem basically is that a fiber product of smooth schemes is not in general smooth and if we do not allow blow-ups to be coverings in our topology a non-smooth scheme can not be covered by smooth ones.

Note that Lemma 3.6 implies in particular that the functor $\pi^{*}$ takes sheaves of abelian groups to sheaves of abelian groups. In an abuse of notation, for any presheaf $F$ on $S m / k$, we denote by $F_{c d h}$ the sheaf $\pi^{*}\left(F_{Z a r}\right)$ where $F_{Z a r}$ is the sheaf in Zariski topology on $S m / k$ associated to $F$.

The results of the rest of this section are not used anywhere in this paper but in the definition of motivic cohomology with compact supports in Section 9. We are going to show that for any sheaf of abelian groups (and more generally any complex of abelian groups) $F$ on $(S c h / k)_{c d h}$ one can define cdh-cohomology with compact supports with coefficients in $F$ which satisfy all the standard properties.

Let us recall that for any scheme $X$ of finite type over $k$ we denote by $\mathbf{Z}(X)$ the presheaf of abelian groups freely generated by the sheaf of sets 
represented by $X$ on $S c h / k$. The universal property of freely generated sheaves implies immediately that for any cdh-sheaf of abelian groups $F$ on $S c h / k$ there are canonical isomorphisms:

$$
H_{c d h}^{i}(X, F)=\operatorname{Ext}^{i}\left(\mathbf{Z}_{c d h}(X), F\right)
$$

where the groups on the right hand side are Ext-groups in the abelian category of cdh-sheaves on $S c h / k$. More generally for any complex of sheaves $K$ one has:

$$
\mathbf{H}_{c d h}^{i}(X, K)=\operatorname{Hom}\left(\mathbf{Z}_{c d h}(X), K[i]\right)
$$

where groups on the left hand side are hypercohomology groups of $X$ with coefficients in $K$ and groups on the right hand side are morphisms in the derived category of cdh-sheaves on $S c h / k$.

Note that for any connected scheme $U$ the group $\mathbf{Z}(X)(U)$ can be described as the free abelian group generated by closed subschemes $Z$ in $X \times U$ such that the projection $Z \rightarrow U$ is an isomorphism. We denote for any $U$ by $\mathbf{Z}^{c}(X)(U)$ the free abelian group generated by closed subschemes $Z$ in $X \times U$ such that the projection $Z \rightarrow U$ is an open embedding. One can easily see that $\mathbf{Z}^{c}(X)(-)$ is a presheaf of abelian groups on $S c h / k$. We call it the presheaf of abelian groups freely generated by $X$ with compact supports. If $X$ is a proper scheme then for any topology $t$ such that disjoint unions are t-coverings we have $\mathbf{Z}_{t}(X)=\mathbf{Z}_{t}^{c}(X)$. The following proposition summarizes elementary properties of sheaves $\mathbf{Z}_{c d h}^{c}(X)$ in cdh-topology.

Proposition 3.8 Let $k$ be a field. One has:

1. The sheaves $\mathbf{Z}_{c d h}^{c}(X)$ are covariantly functorial with respect to proper morphisms $X_{1} \rightarrow X_{2}$.

2. The sheaves $\mathbf{Z}_{c d h}^{c}(X)$ are contravariantly functorial with respect to open embeddings $X_{1} \rightarrow X_{2}$.

3. Let $j: U \rightarrow X$ be an open embedding and $i: X-U \rightarrow X$ be the corresponding closed embedding. Then the following sequence of cdhsheaves is exact:

$$
0 \rightarrow \mathbf{Z}_{c d h}^{c}(X-U) \stackrel{i_{*}}{\rightarrow} \mathbf{Z}_{c d h}^{c}(X) \stackrel{j^{*}}{\rightarrow} \mathbf{Z}_{c d h}^{c}(U) \rightarrow 0
$$


Proof: The first two statements are obvious. To prove the last one note first that the sequence of presheaves

$$
0 \rightarrow \mathbf{Z}^{c}(X-U) \stackrel{i_{*}}{\rightarrow} \mathbf{Z}^{c}(X) \stackrel{j^{*}}{\rightarrow} \mathbf{Z}^{c}(U)
$$

is exact. Since the functor of associated sheaf is exact it implies that we have only to show that $j_{*}$ is a surjection of cdh-sheaves. Let $Y$ be a scheme of finite type over $k$ and $Z$ be an element of $\mathbf{Z}^{c}(U)(Y)$. It is sufficient to show that there is a cdh-covering $p: Y^{\prime} \rightarrow Y$ such that $Z \times_{Y} Y^{\prime}$ as an element of $\mathbf{Z}^{c}(U)\left(Y^{\prime}\right)$ belongs to the image of the homomorphism $\mathbf{Z}^{c}(X)\left(Y^{\prime}\right) \rightarrow$ $\mathbf{Z}^{c}(U)\left(Y^{\prime}\right)$. It follows trivially from the platification theorem [21, Th. 2.2.2], [17] and definition of cdh-topology.

Corollary 3.9 Let $i: X \rightarrow \bar{X}$ be an open embedding of a scheme $X$ over $k$ to a proper scheme $\bar{X}$ over $k$. Then one has a short exact sequence of the form:

$$
0 \rightarrow \mathbf{Z}_{c d h}(\bar{X}-X) \rightarrow \mathbf{Z}_{c d h}(\bar{X}) \rightarrow \mathbf{Z}_{c d h}^{c}(X) \rightarrow 0 .
$$

For any complex of cdh-sheaves $K$ we define the hypercohomology groups $\mathbf{H}_{c}^{i}(X, K)$ of $X$ with compact supports with coefficients in $K$ as the groups of morphisms $\operatorname{Hom}\left(\mathbf{Z}_{c d h}^{c}(X), K[i]\right)$ in the derived category of cdh-sheaves on $S c h / k$. Proposition 3.8 and Corollary 3.9 imply immediately that these groups satisfy standard properties of (hyper-)cohomology with compact supports.

\section{Bivariant cycle cohomology.}

Denote by $\Delta^{n}$ the affine scheme

$$
\Delta^{n}=\operatorname{Spec}\left(k\left[x_{0}, \ldots, x_{n}\right] / \sum x_{i}=1\right)
$$

over $k$. We consider it as an algebro-geometrical analog of n-dimesnional simplex. Proceeding exactly as in topological situation one can define boundary and degeneracy morphisms

$$
\begin{aligned}
\partial_{i}^{n}: \Delta^{n-1} & \rightarrow \Delta^{n} \\
\sigma_{i}^{n}: \Delta^{n+1} & \rightarrow \Delta^{n}
\end{aligned}
$$


such that $\Delta^{\bullet}=\left(\Delta^{n}, \partial_{i}^{n}, \sigma_{i}^{n}\right)$ is a cosimplicial object in the category $S m / k$.

For any presheaf $F$ on $S c h / k$, the category of schemes of finite type over $k$, and any such $k$-scheme $U$, consider the simplicial set $F\left(\Delta^{\bullet} \times U\right)$. If $F$ is a presheaf of abelian groups it is a simplicial abelian group and we denote by $\underline{C}_{*}(F)(U)$ the corresponding complex of abelian groups (i.e. $\underline{C}_{n}(F)(U)=F\left(\Delta^{n} \times U\right)$ and the differencial is given by alternated sums of homomorphisms $\left.F\left(\partial_{i}^{n} \times I d_{U}\right)\right)$. Clearly, $\underline{C}_{*}(F)$ is a complex of presheaves on $S c h / k$, which we call the singular simplicial complex of $F$. We denote by

$$
\underline{h}_{i}(F)=H^{-i}\left(\underline{C}_{*}(F)\right)
$$

the cohomology presheaves of $\underline{C}_{*}(F)$.

If $F$ is a presheaf defined on the full subcategory $S m / k$ of schemes smooth over $k$ and if $U$ is a smooth scheme, then we shall employ the same notation and terminology for the same constructions applied to $F$.

The following lemma shows that the presheaves of the form $\underline{h}_{i}(-)$ are homotopy invariant.

Lemma 4.1 Let $F$ be a presheaf on $S c h / k$ (respectively, Sm/k). Then for any scheme $U$ of finite type over $k$ (resp., smooth of finite type over $k$ ), and any $i \in \mathbf{Z}$ the morphism $\underline{h}_{i}(F)(U) \rightarrow \underline{h}_{i}(F)\left(U \times \mathbf{A}^{1}\right)$ induced by the projection $U \times \mathbf{A}^{1} \rightarrow U$ is an isomorphism.

Proof: Denote by $i_{0}, i_{1}: U \rightarrow U \times \mathbf{A}^{1}$ the closed embeddings $I d_{U} \times\{0\}$ and $I d_{U} \times\{1\}$ respectively. Let us show first that the morphisms

$$
\begin{aligned}
& i_{0}^{*}: \underline{h}_{i}(F)\left(U \times \mathbf{A}^{1}\right) \rightarrow \underline{h}_{i}(F)(U) \\
& i_{1}^{*}: \underline{h}_{i}(F)\left(U \times \mathbf{A}^{1}\right) \rightarrow \underline{h}_{i}(F)(U)
\end{aligned}
$$

coincide. It is sufficient to prove that the corresponding morphisms of complexes of abelian groups $\underline{C}_{*}(F)\left(U \times \mathbf{A}^{1}\right) \rightarrow \underline{C}_{*}(F)(U)$ are homotopic.

Define a homomorphism

$$
s_{n}: F\left(U \times \mathbf{A}^{1} \times \Delta^{n}\right) \rightarrow F\left(U \times \Delta^{n+1}\right)
$$

by the formula

$$
s_{n}=\sum_{i=0}^{n}(-1)^{i}\left(I d_{U} \times \psi_{i}\right)^{*}
$$


where $\psi_{i}: \Delta^{n+1} \rightarrow \Delta^{n} \times \mathbf{A}^{1}$ is the linear isomorphism taking $v_{j}$ to $v_{j} \times 0$ if $j \leq i$ or to $v_{j-1} \times 1$ if $j>i$ (here $v_{j}=(0, \ldots, 1, \ldots, 0)$ is the $\mathrm{j}$-th vertex of $\Delta^{n+1}\left(\right.$ resp. $\left.\left.\Delta^{n}\right)\right)$. A staraightforward computation shows that $s d+d s=$ $i_{1}^{*}-i_{0}^{*}$.

Consider now the morphism

$$
I d_{U} \times \mu: U \times \mathbf{A}^{1} \times \mathbf{A}^{1} \rightarrow U \times \mathbf{A}^{1}
$$

where $\mu: \mathbf{A}^{1} \times \mathbf{A}^{1} \rightarrow \mathbf{A}^{1}$ is given by multiplication of functions. Applying the previous result to the embeddings

$$
i_{0}, i_{1}: U \times \mathbf{A}^{1} \rightarrow\left(U \times \mathbf{A}^{1}\right) \times \mathbf{A}^{1}
$$

we conclude that the homomorphism

$$
\underline{h}_{i}(F)\left(U \times \mathbf{A}^{1}\right) \rightarrow \underline{h}_{i}(F)\left(U \times \mathbf{A}^{1}\right)
$$

induced by the composition

$$
U \times \mathbf{A}^{1} \stackrel{p r_{1}}{\rightarrow} U \stackrel{i_{0}}{\rightarrow} U \times \mathbf{A}^{1}
$$

is the identity homomorphism which implies immediately the assertion of the lemma.

We will use frequently (without explicit mention) the following elementary fact. Let

$$
0 \rightarrow F \rightarrow G \rightarrow H \rightarrow 0
$$

be a short exact sequence of presheaves of abelian groups on either $S c h / k$ or $S m / k$. Then the corresponding sequence of complexes of presheaves

$$
0 \rightarrow \underline{C}_{*}(F) \rightarrow \underline{C}_{*}(G) \rightarrow \underline{C}_{*}(H) \rightarrow 0
$$

is exact. In particular there is a long exact sequence of presheaves of the form

$$
\ldots \rightarrow \underline{h}_{i}(F) \rightarrow \underline{h}_{i}(G) \rightarrow \underline{h}_{i}(H) \rightarrow \underline{h}_{i-1}(F) \rightarrow \ldots
$$

For any smooth scheme $U$ over $k$ and a scheme of finite type $X$ over $k$ consider the abelian groups $\underline{h}_{i}\left(z_{\text {equi }}(X, r)\right)(U)$. These groups are contravariantly functorial with respect to $U$ and covariantly (resp. contravariantly) functorial with respect to proper morphisms (resp. flat equidimensional morphisms) in $X$. The following theorem summarizes most of the known results which relate the groups $\underline{h}_{i}\left(z_{\text {equi }}(X, r)\right)(U)$ to other theories. 
Theorem 4.2 Let $k$ be a field.

1. For any scheme $X$ over $k$ the group $\underline{h}_{0}\left(z_{\text {equi }}(X, r)(\operatorname{Spec}(k))\right.$ is canonically isomorphic to the group $A_{r}(X)$ of cycles of dimension $r$ on $X$ modulo rational equivalence.

2. For any equidimensional affine scheme $X$ over $k$ and any $r \geq 0$ there are canonical isomorphisms

$$
\underline{h}_{i}\left(z_{\text {equi }}(X, r)\right)(\operatorname{Spec}(k)) \rightarrow C H^{\operatorname{dim}(X)-r}(X, i)
$$

where the groups on the right hand side are higher Chow groups of $X$ defined in [1].

3. Assume $k$ is of characteristic 0 and $X$ is a normal equidimensional scheme of pure dimension $n$ or that $k$ is a perfect field and that $X$ is a normal affine scheme of pure dimension $n$. Then the groups $\underline{h}_{i}\left(z_{\text {equi }}(X, n-1)\right)(\operatorname{Spec}(k))$ are of the form:

$$
\underline{h}_{i}\left(z_{\text {equi }}(X, n-1)\right)(\operatorname{Spec}(k))= \begin{cases}A_{n-1}(X) & \text { for } i=0 \\ \mathcal{O}^{*}(X) & \text { for } i=1 \\ 0 & \text { for } i \neq 0,1\end{cases}
$$

4. The groups $\underline{h}_{0}\left(z_{\text {equi }}\left(\mathbf{A}^{n}, 0\right)\right)(\operatorname{Spec}(k))$ are isomorphic to the Milnor $K$ groups $K_{n}^{M}(k)$.

5. If $k$ is an algebraically closed field which admits resolution of singularities, $X$ is a smooth scheme over $k$ of dimension $m$, and $n \neq 0$ is an integer prime to char $(k)$, the groups $\underline{h}_{i}\left(z_{\text {equi }}(X, 0) \otimes \mathbf{Z} / n \mathbf{Z}\right)$ are isomorphic to the etale cohomology groups $H^{2 m-i}(X, \mathbf{Z} / n \mathbf{Z})$.

6. If $k=\mathbf{C}$, then for any quasi-projective variety $X$ (i.e., reduced, irreducible $\mathbf{C}$-scheme) the groups $\underline{h}_{i}\left(z_{\text {equi }}(X, r) \otimes \mathbf{Z} / n \mathbf{Z}\right)$ are isomorphic to the corresponding Lawson homology with finite coefficients.

\section{Proof:}

1. Elementary (see for instance [5]). 
2. Note that for any equidimensional scheme $X$ over $k$ and any $r$ the complex $\underline{C}_{*}\left(z_{\text {equi }}(X, r)\right)(\operatorname{Spec}(k))$ which computes the groups $\underline{h}_{i}\left(z_{\text {equi }}(X, r)(\operatorname{Spec}(k))\right.$ can be considered as a subcomplex in the Bloch complex $\mathcal{Z}^{\operatorname{dim}(X)-r}(X, *)$ which computes the higher Chow groups of $X$ (see [1],[6]). The fact that this morphism is a quasi-isomorphism for affine schemes $X$ was proven by A. Suslin (see [20])2.

3. In [15], $C H^{1}(X, i)$ was computed for $X$ a normal scheme of pure dimension $n$ over a perfect field thus, the assertion for $X$ affine and normal follows from [15]. In [5], $z_{\text {equi }}(X, n-1)_{h}$ was computed for $X$ arbitrary and the computation agrees with that of assertion (3) for $X$ normal; by Remarks 4.6, 5.10, this implies assertion (3) provided $k$ has characteristic 0 .

4. This follows from part (2), the homotopy invariance property for higher Chow groups, and $[1]^{3}$.

5. This was proven in [22].

6. This was proven in [22] for $X$ projective and the extended to quasiprojective $X$ in [5].

One would like to consider $\underline{h}_{i}(Z(X, r))(U)$ as a natural bivariant generalization of groups $A_{r}(X)$ (and more generaly of the higher Chow groups of $X)$. In fact, we shall consider a somewhat more sophisticated construction (cf. Definition 2.11) in order to enable the Mayer-Vietoris property with respect to the first argument of our bivariant theory.

Note that functoriality of the groups $\underline{h}_{i}(Z(X, r))(U)$ shows that they behave as a cohomology theory with respect to the first argument $(U)$ and as a Borel-Moore homology theory with respect to the second $(X)$. Thus, one would expect that other properties of these classes of theories should hold for the groups $\underline{h}_{i}\left(z_{\text {equi }}(X, r)\right)(U)$. In particular, there should exist a localization long exact sequence with respect to $X$ and a Mayer-Vietoris long exact

\footnotetext{
${ }^{2}$ The proof is based on an elementary moving technique and does not use any of the advanced properties of either higher Chow groups or groups $\underline{h}_{i}\left(z_{\text {equi }}(X, r)\right)(-)$.

${ }^{3}$ Another way to prove it (in the case of a perfect field $k$ ) is to use Mayer-Vietoris sequence in Suslin homology proven in [23] together with direct computation of Suslin homology of $\left(\mathbf{A}^{1}-\{0\}\right)^{n}$.
} 
sequence with respect to $U$. We will show below that this is indeed true for quasi-projective schemes $U$ if $k$ admits resolution of singularities. It turns out though that it is inconvenient to work with the groups $\underline{h}_{i}\left(z_{\text {equi }}(X, r)\right)(U)$ directly. Instead we define for all schemes of finite type $Y, X$ over $k$ the $b i$ variant cycle cohomology groups $A_{r, i}(Y, X)$ of $Y$ with coefficients in r-cycles on $X$ as certain hypercohomology groups of $Y$ which automatically gives us most of the good properties with respect to $Y$. We will show then that for a field $k$ which admits resolution of singularities and for a smooth quasiprojective $k$-scheme $U$ the groups $A_{r, i}(U, X)$ are canonically isomorphic to the groups $\underline{h}_{i}\left(z_{\text {equi }}(X, r)(U)\right)$.

Definition 4.3 Let $X, Y$ be schemes of finite type over a field $k$ and $r \geq 0$ be an integer. The bivariant cycle cohomology groups of $Y$ with coefficients in cycles on $X$ are the groups

$$
A_{r, i}(Y, X)=\mathbf{H}_{c d h}^{-i}\left(Y,\left(\underline{C}_{*}\left(z_{\text {equi }}(X, r)\right)_{c d h}\right) .\right.
$$

We will also use the notation $A_{r, i}(X)$ for the groups $A_{r, i}(\operatorname{Spec}(k), X)$.

It follows immediately from this definition that the groups $A_{r, i}(Y, X)$ are contravariantly functorial with respect to $Y$ and covariantly (resp. contravariantly) functorial with respect to proper morphisms (resp. flat equidimensional morphisms) in $X$. It is also clear that the groups $A_{r, i}(X)$ coincide with the groups $\underline{h}_{i}\left(z_{\text {equi }}(X, r)\right)(\operatorname{Spec}(k))$. In particular, Theorem 4.2(2) implies that for affine equidimensional schemes $X$ the groups $A_{r, i}(X)$ are isomorphic to the corresponding higher Chow groups.

Since open coverings are cdh-coverings the following proposition is a trivial corollary of our definition.

Proposition 4.4 Let $X, Y$ be schemes of finite type over a field $k$. Let further $Y=U_{1} \cup U_{2}$ be a Zariski open covering of $Y$. Then for any $r \geq 0$ there is a canonical long exact sequence of the form:

$$
\begin{gathered}
\ldots \rightarrow A_{r, i}(Y, X) \rightarrow A_{r, i}\left(U_{1}, X\right) \oplus A_{r, i}\left(U_{2}, X\right) \rightarrow A_{r, i}\left(U_{1} \cap U_{2}, X\right) \rightarrow \\
\rightarrow A_{r, i-1}(Y, X) \rightarrow \ldots
\end{gathered}
$$

Since abstract blow-ups are cdh-coverings (definition 3.2) we immediately obtain the following blow-up exact sequence with respect to the first argument. 
Proposition 4.5 Let $p: Y^{\prime} \rightarrow Y$ be a proper morphism of schemes of finite type over $k$ and $Z \subset Y$ be a closed subscheme of $Y$ such that the morphism $p^{-1}(Y-Z) \rightarrow Y-Z$ is an isomorphism. Then for any scheme of finite type $X$ over $k$ and any $r \geq 0$ there is a canonical long exact sequence of the form

$$
\begin{gathered}
\ldots \rightarrow A_{r, i}(Y, X) \rightarrow A_{r, i}\left(Y^{\prime}, X\right) \oplus A_{r, i}(Z, X) \rightarrow A_{r, i}\left(p^{-1}(Z), X\right) \rightarrow \\
\rightarrow A_{r, i-1}(Y, X) \rightarrow \ldots
\end{gathered}
$$

Remark 4.6 The first definition of sheaves of relative cycles similar to $z_{\text {equi }}(X, r)_{c d h}$ was given in [6] together with a definition of the corresponding "naive" bivariant cycle cohomology groups. As was shown in [5] the presheaves defined in [6] are isomorphic to the sheaves $z_{\text {equi }}(X, r)_{h}$ in the h-topology (see [25] or [22]) on $S c h / k$ associated with $z_{\text {equi }}(X, r)_{c d h}$.

The cdh-sheaves $z_{\text {equi }}(X, r)_{c d h}$ were considered in [21] where they are denoted by $z(X, r)$ (see [21, Th. 4.2.9(2)]). As was shown in [21], the canonical morphism

$$
z_{\text {equi }}(X, r)_{c d h} \rightarrow z_{\text {equi }}(X, r)_{h}
$$

is a monomorphism and it becomes an isomorphism after tensoring with $\mathbf{Z}[1 / p]$ where $p$ is the exponential characteristic of $k$. In particular for a quasi-projective scheme $X$ over a field of characteristic zero, the sheaves $z_{\text {equi }}(X, r)_{c d h}$ are isomorphic to the presheaves constructed in [6].

One can show easily (we will not use this fact in the paper) that for any scheme of finite type $X$ over $k$ the restriction of the cdh-sheaf $z_{\text {equi }}^{e f f}(X, r)_{c d h}$ to $S m / k$ coincides with $z_{\text {equi }}^{e f f}(X, r)$. On the other hand, the corresponding statement for $z_{\text {equi }}(X, r)$ is false: the natural homomorphism $z_{\text {equi }}(X, r)(V) \rightarrow$ $z_{\text {equi }}(X, r)_{c d h}(V)$ is a monomorphism but not in general an epimorphism. Nevertheless, it turns out that this difference is insignificant from the point of view of bivariant cycle cohomology. More precisely, it will be shown in the next section (see remark 5.10) that for any scheme of finite type $X$ over $k$ the groups $\underline{h}_{i}\left(z_{\text {equi }}(X, r)\right)(\operatorname{Spec}(k))$ and the groups $\underline{h}_{i}\left(z_{\text {equi }}(X, r)_{\text {cdh }}\right)(\operatorname{Spec}(k))$ are isomorphic.

\section{Pretheories.}

One of our main technical tools is the "theory of pretheories" developed in [23]. Informally speaking, a pretheory is a presheaf of abelian groups 
on $S m / k$ which has transfers with respect to finite coverings (the precise definition is given below). We will show below how to define a natural structure of pretheory on presheaves $z_{\text {equi }}(X, r)$. The main reason why this class of presheaves is important for us is that the singular simplicial complexes $\underline{C}_{*}(F)$ of pretheories $F$ behave "nicely"; in particular we obtain the localization theorem (5.11) in our theory.

Let $U$ be a smooth scheme over $k$ and $C \rightarrow U$ be a smooth curve over $U$. Denote by $c_{e q u i}(C / U, 0)$ the free abelian group generated by integral closed subschemes in $C$ which are finite over $U$ and dominant over an irreducible component of $U$. Any such subscheme is flat over $U$. In particular for a morphism $f: U^{\prime} \rightarrow U$ there is defined a homomorphism $\operatorname{cycl}(f): c_{\text {equi }}(C / U, 0) \rightarrow c_{\text {equi }}\left(C \times_{U} U^{\prime} / U^{\prime}, 0\right)$. For any section $s: U \rightarrow C$ of the projection $C \rightarrow U$ its image is an element in $c_{\text {equi }}(C / U, 0)$ which we denote by $[s]$. A pretheory (with values in the category of abelian groups) on the category $S m / k$ is a presheaf $F$ of abelian groups together with a family of homomorphisms $\phi_{C / U}: c_{\text {equi }}(C / U, 0) \rightarrow \operatorname{Hom}(F(C), F(U))$ given for all smooth curves $C \rightarrow U$ over smooth schemes over $k$ and satisfying the following conditions:

1. For any smooth schemes $U_{1}, U_{2}$ over $k$ the canonical homomorphism $F\left(U_{1} \amalg U_{2}\right) \rightarrow F\left(U_{1}\right) \oplus F\left(U_{2}\right)$ is an isomorphism.

2. For a section $s: U \rightarrow C$ one has $\phi_{C / U}([s])=F(s)$.

3. For a morphism $f: U^{\prime} \rightarrow U$, an element $a \in F(C)$ and an element $\mathcal{Z}$ in $c_{\text {equi }}(C / U, 0)$ one has

$$
F(f)\left(\phi_{C / U}(\mathcal{Z})(a)\right)=\phi_{C \times_{U} U^{\prime} / U^{\prime}}(\operatorname{cycl}(f))(\mathcal{Z})\left(F\left(f \times_{U} C\right)(a)\right) .
$$

A morphism of pretheories is a morphism of presheaves which is consistent with the structures of pretheories in the obvious way.

Note that the category of pretheories is abelian and the forgetful functor from this category to the category of presheaves of abelian groups is exact. In particular for any pretheory $F$ on $S m / k$ the cohomology presheaves $\underline{h}_{*}(F)$ are pretheories.

A pretheory $F$ is called homotopy invariant if for any smooth scheme $U$ over $k$ the homomorphism $F(U) \rightarrow F\left(U \times \mathbf{A}^{1}\right)$ induced by the projection $U \times \mathbf{A}^{1} \rightarrow U$ is an isomorphism. Note that by lemma 4.1 for any pretheory $F$ the pretheories $\underline{h}_{i}(F)$ are homotopy invariant. 
Theorem 5.1 Let $k$ be a perfect field and $F$ be a homotopy invariant pretheory on $S m / k$. Then one has:

1. For any $i \geq 0$ the presheaf $H_{Z a r}^{i}\left(-, F_{Z a r}\right)$ has a canonical structure of a homotopy invariant pretheory. In particular $F_{Z a r}$ is a homotopy invariant pretheory and for any smooth scheme $U$ over $k$ the homomorphisms

$$
H_{Z a r}^{i}\left(U, F_{Z a r}\right) \rightarrow H_{Z a r}^{i}\left(U \times \mathbf{A}^{1}, F_{Z a r}\right)
$$

are isomorphisms.

2. For any smooth scheme $U$ over $k$ and any $i \geq 0$ the canonical homomorphism

$$
H_{Z a r}^{i}\left(U, F_{Z a r}\right) \rightarrow H_{N i s}^{i}\left(U, F_{N i s}\right)
$$

is an isomorphism. In particular $F_{Z a r}=F_{N i s}$.

Proof: See [23, Th. 4.27] for the first part and [23, Th. 5.7] for the second.

This theorem implies easily the following important criterion of vanishing for sheaves $\underline{h}_{i}(F)_{Z a r}$ if $F$ is a pretheory.

Proposition 5.2 Let $k$ be a perfect field and $F$ be a pretheory on $S m / k$. Then the following conditions are equivalent.

1. The sheaves $\underline{h}_{i}(F)_{\text {Zar }}$ are zero for all $i \leq n$.

2. For any homotopy invariant pretheory $G$ and any $i<n$, one has

$$
\operatorname{Ext}^{i}\left(F_{N i s}, G_{N i s}\right)=0
$$

(here Ext ${ }^{i}(-,-)$ is the Ext-group in the category of Nisnevich sheaves on $S m / k)$.

Proof: See [23, Th. 5.9].

The key to our localization theorem for bivariant cycle homology is the following statement (cf Theorem 5.5): if $F$ is a pretheory such that $F_{c d h}=0$ and if $k$ admits resolution of singularities, then $\underline{h}_{i}(F)_{Z a r}=0$. We observe that if we the weaken the condition $F_{c d h}=0$ to $F_{N i s}=0$, then this follows 
immediately from Proposition 5.2. To obtain the stronger result we need, we first require the following two lemmas.

Let us recall that for a smooth scheme $U$ over $k$ we denote by $\mathbf{Z}(U)$ the presheaf of abelian groups on $S m / k$ freely generated by the presheaf of sets represented by $U$. We will use the following lemma.

Lemma 5.3 Let $k$ be a perfect field, $U$ be a smooth scheme over $k$ and $Z \subset U$ be a smooth closed subscheme of $U$. Denote by $p: U_{Z} \rightarrow U$ the blow-up of $U$ with center in $Z$. Then for any homotopy invariant pretheory $G$ on $S m / k$ and any $i \geq 0$ one has:

$$
\operatorname{Ext}^{i}\left(\operatorname{coker}\left(\mathbf{Z}\left(U_{Z}\right) \rightarrow \mathbf{Z}(U)\right)_{N i s}, G_{N i s}\right)=0 .
$$

Proof: See [23, Prop. 5.21].

The main technical ingredient for the proof of Theorem 5.5 is isolated in the following lemma.

Lemma 5.4 Let $k$ be a field which admits resolution of singularities and let $F$ be a Nisnevich sheaf of abelian groups on $S m / k$ such that $F_{c d h}=0$. Then for any homotopy invariant pretheory $G$ and any $i \geq 0$ one has

$$
\operatorname{Ext}^{i}\left(F, G_{N i s}\right)=0 .
$$

Proof: In view of Theorem 5.1 we may assume that $G_{N i s}=G$, i.e. that $G$ is a sheaf in Nisnevich topology. We use induction by $i$. Since our statement is trivial for $i<0$ we may assume that for any sheaf $F$ satisfying the condition of the proposition and any $j<i$ one has $\operatorname{Ext}^{j}(F, G)=0$.

Let $U$ be a smooth scheme over $k$ and $p: U^{\prime} \rightarrow U$ be a morphism which is a composition of $n$ blow-ups with smooth centers. Let us show that $\operatorname{Ext}^{i}\left(\operatorname{coker}(\mathbf{Z}(p))_{N i s}, G\right)=0$. Let $p=p_{0} \circ p_{1}$ where $p_{0}$ is a blowup with a smooth center and $p_{1}$ is a composition of $n-1$ blow-ups with smooth centers. By induction on $n$ and Lemma 5.3 we may assume that $\operatorname{Ext}^{i}\left(\operatorname{coker}\left(\mathbf{Z}\left(p_{\epsilon}\right)\right)_{N i s}, G\right)=0$ for $\epsilon=0,1$. We have an exact sequence of presheaves

$$
0 \rightarrow \Psi \rightarrow \operatorname{coker}\left(\mathbf{Z}\left(p_{1}\right)\right) \rightarrow \operatorname{coker}(\mathbf{Z}(p)) \rightarrow \operatorname{coker}\left(\mathbf{Z}\left(p_{0}\right)\right) \rightarrow 0
$$

We rewrite this exact sequence as two short exact sequences

$$
0 \rightarrow \Psi \rightarrow \operatorname{coker}\left(\mathbf{Z}\left(p_{1}\right)\right) \rightarrow \Psi^{\prime} \rightarrow 0
$$




$$
0 \rightarrow \Psi^{\prime} \rightarrow \operatorname{coker}(\mathbf{Z}(p)) \rightarrow \operatorname{coker}\left(\mathbf{Z}\left(p_{0}\right)\right) \rightarrow 0
$$

Since $(\Psi)_{c d h}=0$ the long exact sequences of Ext-groups associated with two short exact sequences above together with the induction assumption and Lemma 5.3 imply now that $\operatorname{Ext}^{i}\left(\operatorname{coker}(\mathbf{Z}(p))_{N i s}, G\right)=0$.

Let now $F$ be any Nisnevich sheaf such that $F_{c d h}=0$. Consider the epimorphism

$$
\oplus \phi_{\alpha}: \oplus \mathbf{Z}\left(U_{\alpha}\right) \rightarrow F
$$

where the sum is taken over all pairs of the form $\left(U_{\alpha}, \phi_{\alpha}\right)$ where $U_{\alpha}$ is a smooth scheme over $k$ and $\phi_{\alpha} \in F\left(U_{\alpha}\right)$. It follows from resolution of singularities and Lemma 3.3 that for any smooth scheme $U_{\alpha}$ over $k$ and any section $\phi_{\alpha} \in$ $F\left(U_{\alpha}\right)$ there is a sequenece of blow-ups with smooth centers $p_{\alpha}: U_{\alpha}^{\prime} \rightarrow U_{\alpha}$ such that $p_{\alpha}^{*}\left(\phi_{\alpha}\right)=0$. Thus our epimorphism can be factorized through an epimorphism $\Psi \rightarrow F$ where $\Psi=\oplus_{\alpha} \operatorname{coker}\left(\mathbf{Z}\left(p_{\alpha}\right)\right)$. Let $\Psi_{0}$ be the kernel of this epimorphism. Then $\left(\Psi_{0}\right)_{c d h}=0$. Since we have already proven that $\operatorname{Ext}^{i}\left(\Psi_{N i s}, G\right)=0$ we conclude that $\operatorname{Ext}^{i}\left(F_{N i s}, G\right)=0$ from the induction assumption and the long exact sequence of Ext-groups associated with the short exact sequence of presheaves

$$
0 \rightarrow \Psi_{0} \rightarrow \Psi \rightarrow F \rightarrow 0 .
$$

Theorem 5.5 Let $k$ be a field which admits resolution of singularities and $F$ be a pretheory over $k$.

1. For any smooth scheme $U$ over $k$, one has canonical isomorphisms

$$
\mathbf{H}_{c d h}^{i}\left(U, \underline{C}_{*}(F)_{c d h}\right)=\mathbf{H}_{Z a r}^{i}\left(U, \underline{C}_{*}(F)_{Z a r}\right) .
$$

2. If $F_{c d h}=0$, then $\underline{C}_{*}(F)_{Z a r}$ is acyclic.

3. For any scheme of finite type $X$ over $k$, the projection $X \times \mathbf{A}^{1} \rightarrow X$ induces isomorphisms

$$
\mathbf{H}_{c d h}^{i}\left(X, \underline{C}_{*}(F)_{c d h}\right) \rightarrow \mathbf{H}_{c d h}^{i}\left(X \times \mathbf{A}^{1}, \underline{C}_{*}(F)_{c d h}\right) .
$$

Proof: To prove part (1) of the theorem it is sufficient in view of hypercohomology spectral sequence and Theorem 5.1(2) to show that for any homotopy invariant pretheory $G$ one has:

$$
H_{c d h}^{i}\left(U, G_{c d h}\right)=H_{N i s}^{i}\left(U, G_{N i s}\right)
$$


(we then apply it to $G=\underline{h}_{n}(F)$ ).

Let $\mathcal{U}$ be a cdh-hypercovering of $U$. We say that it is smooth if all schemes $U_{i}$ are smooth. Resolution of singularities guarantees that any cdhhypercovering of $U$ has a smooth refiniment. For any smooth hypercovering $\mathcal{U}$ denote by $\mathbf{Z}(\mathcal{U})$ the complex of presheaves on $S m / k$ with terms $\mathbf{Z}\left(U_{i}\right)$ and differentials given by alternated sums of morphisms induced by boundary morphisms of the simplicial scheme $\mathcal{U}$. The standard description of cohomology in terms of hypercoverings gives us for any presheaf $G$ a canonical isomorphism

$$
H_{c d h}^{i}\left(U, G_{c d h}\right)=\lim _{\mathcal{U}}\left(\operatorname{Hom}\left(\mathbf{Z}(\mathcal{U})_{N i s}, G_{N i s}[i]\right)\right)
$$

where the limit on the right hand side is taken over all smooth cdh-hypercoverings of $U$ and $\operatorname{Hom}(-,-)$ refers to morphisms in the derived category of the category of sheaves of abelian groups on $(\mathrm{Sm} / \mathrm{k})_{\mathrm{Nis}}$ (note that we could replace in this isomorphism $N i s$ by any topology which is weaker then the cdh-toplogy). There is a canonical morphism of complexes $\mathbf{Z}(\mathcal{U}) \rightarrow$ $\mathbf{Z}(U)$. Denote its cone by $K_{\mathcal{U}}$. By definition of a hypecovering the complex of cdh-sheaves $\left(K_{\mathcal{U}}\right)_{c d h}$ is quasi-isomorphic to zero, i.e. for any $j \in \mathbf{Z}$ one has $\underline{H}^{j}\left(K_{\mathcal{U}}\right)_{c d h}=0$ where $\underline{H}^{j}\left(K_{\mathcal{U}}\right)$ are cohomology presheaves of $K_{\mathcal{U}}$. Thus by Lemma 5.4 for a homotopy invariant pretheory $G$ the homomorphisms

$$
\operatorname{Hom}\left(\mathbf{Z}(U)_{N i s}, G_{N i s}[i]\right) \rightarrow \operatorname{Hom}\left(\mathbf{Z}(\mathcal{U})_{N i s}, G_{N i s}[i]\right)
$$

are isomorphisms for all $i \in \mathbf{Z}$. Since the left hand side groups are canonically isomorphic to $H_{N i s}^{i}\left(U, G_{N i s}\right)$ it proves part (1) of the theorem.

Let now $F$ be a pretheory such that $F_{c d h}=0$. We want to show that $\underline{C}_{*}(F)_{Z a r}$ is quasi-isomorphic to zero. By Theorem 5.1(2) it is sufficient to show that $\underline{C}_{*}(F)_{N i s}$ is quasi-isomiorphic to zero. Suppose that $\underline{h}_{n}(F)_{N i s} \neq 0$ for some $n$. We may assume that $\underline{h}_{i}(F)_{N i s}=0$ for all $i<n$. Then there is a non trivial morphism in the derived category $\left.\mathcal{D}(S m / k)_{N i s}\right)$ of sheaves on $(S m / k)_{N i s}$ of the form $\underline{C}_{*}(F)_{N i s} \rightarrow \underline{h}_{n}(F)_{N i s}[n]$. The second part of our theorem follows now from 5.4 and lemma below.

Lemma 5.6 Let $F$ be a presheaf on $S m / k$ and $G$ be a homotopy invariant pretheory on $S m / k$. Then for any $n \in \mathbf{Z}$ one has a canonical isomorphism

$$
\operatorname{Hom}_{\left.\mathcal{D}(S m / k)_{N} i s\right)}\left(\underline{C}_{*}(F)_{N i s}, G_{N i s}[n]\right)=\operatorname{Ext}^{n}\left(F_{N i s}, G_{N i s}\right) .
$$


Proof: See proof of [23, Th. 5.9].

To prove the third part of our theorem, we use smooth hypercoverings of $X$ as in the proof of part (1) and apply part (1) and Theorem 5.1(1).

Let $X$ be a scheme of finite type over $k$. We define a structure of pretheory on the presheaf $z_{\text {equi }}(X, r)$ as follows. Let $C \rightarrow U$ be a smooth curve over a smooth scheme over $k, Z$ be an integral closed subscheme of $C$ which belongs to $c_{\text {equi }}(C / U, 0)$ and $\mathcal{W}$ be an element of $z_{\text {equi }}(X, r)(C)$. Denote by $z$ the generic point of $Z$ and consider the specialization $\mathcal{W}_{z}$. Namely, let $\mathcal{O}_{C, z}$ denote the discrete valuation ring obtained by localizing $C$ at $z$ and let $k(z)$ denote its residue field, equal to the field of fractions of $Z$. Then the restriction $W \times_{C} \operatorname{Spec}\left(\mathcal{O}_{C, z}\right)$ of any irreducible component $W \subset X \times C$ of $\mathcal{W}$ is flat over $\operatorname{Spec}\left(\mathcal{O}_{C, z}\right)$ and $\mathcal{W}_{z}$ is defined to be the sum over the irreducible components $W$ of the cycles associated to $W \times_{C} \operatorname{Spec}(k(z))$.

We set $\phi_{C / U}(Z)(\mathcal{W})$ to be the push-forward of the closure of $\mathcal{W}_{z}$ in $Z \times X$ to $U \times X$.

Proposition 5.7 Let $X$ be a scheme of finite type over $k, U$ be a smooth scheme over $k$ and $C / U$ be a smooth curve over $U$. Then one has:

1. For any element $\mathcal{W}$ in $z_{\text {equi }}(X, r)(C)$ the cycle $\phi_{C / U}(Z)(\mathcal{W})$ defined above belongs to $z_{\text {equi }}(X, r)(U)$.

2. The presheaf $z_{\text {equi }}(X, r)$ together with homomorphisms $\phi_{C / U}$ is a pretheory.

Proof: Our homomorphism

$$
\phi_{C / U}: z_{\text {equi }}(X, r)(C) \otimes c_{\text {equi }}(C / U, 0) \rightarrow C y c l(U \times X)
$$

is a particular case of correspondence homomorphisms considered in [21, Section 3.7]. The first statement of the proposition is a particular case of the first statement of [21, Th. 3.7.3].

First two conditions of the definition of pretheory are hold for trivial reasons. The third one is a particular case of the second statement of $[21$, Th. 3.7.3]. 
Proposition 5.8 1. For any proper morphism $p: X^{\prime} \rightarrow X$ the pushforward homomorphism of presheaves

$$
p_{*}: z_{\text {equi }}\left(X^{\prime}, r\right) \rightarrow z_{\text {equi }}(X, r)
$$

is a morphism of pretheories.

2. For any flat equidimensional morphism $f: X^{\prime} \rightarrow X$ the pull-back homomorphism

$$
f^{*}: z_{\text {equi }}(X, r) \rightarrow z_{\text {equi }}\left(X^{\prime}, r+\operatorname{dim}\left(X^{\prime} / X\right)\right)
$$

is a homomorphism of pretheories.

3. For any smooth equidimensional scheme $U$ and any scheme of finite type $X$ over $k$ the duality homomorphisms

$$
\mathcal{D}: z_{\text {equi }}(U, X, r) \rightarrow z_{\text {equi }}(U \times X, r+\operatorname{dim}(U))
$$

are morphisms of pretheories.

Proof: The second and the third assertions are trivial. The first one is a particular case of [21, Prop. 3.7.6].

Proposition 5.9 Let $k$ be a field which admits resolution of singularities and let $X$ be a scheme of finite type over $k$. Then for any scheme $Y$ over $k$ the homomorphisms

$$
A_{r, i}(Y, X) \rightarrow A_{r, i}\left(Y \times \mathbf{A}^{1}, X\right)
$$

induced by the projection are isomorphisms.

Proof: This is a particular case of Theorem 5.5(3) with $F=z_{\text {equi }}(X, r)$.

Remark 5.10 It is easy to show that for any scheme of finite type $X$ over $k$ the restriction of the sheaf $z_{\text {equi }}(X, r)_{c d h}$ to $S m / k$ has a unique structure of pretheory such that the morphism of presheaves on $S m / k$

$$
z_{\text {equi }}(X, r) \rightarrow z_{\text {equi }}(X, r)_{c d h}
$$

is a morphism of pretheories. Applying Theorem 5.5(2) to kernel and cokernel of this morphism we conclude that

$$
\underline{h}_{i}\left(z_{\text {equi }}(X, r)\right)(\operatorname{Spec}(k))=\underline{h}_{i}\left(z_{\text {equi }}(X, r)_{\text {cdh }}\right)(\operatorname{Spec}(k)) .
$$


Observe that Theorem 5.5(2) implies that any sequence of pretheories

$$
0 \rightarrow F \rightarrow G \rightarrow H \rightarrow 0
$$

such that the corresponding sequence of cdh-sheaves

$$
0 \rightarrow F_{c d h} \rightarrow G_{c d h} \rightarrow H_{c d h} \rightarrow 0
$$

is exact gives us an exact triangle of complexes of sheaves in Zariski topology of the form

$$
\underline{C}_{*}(F)_{Z a r} \rightarrow \underline{C}_{*}(G)_{Z a r} \rightarrow \underline{C}_{*}(H)_{Z a r} \rightarrow \underline{C}_{*}(F)_{Z a r}[1]
$$

and in particular a long exact sequence of the corresponding groups $\underline{h}_{i}(-)(\operatorname{Spec}(k))$.

By Lemma 3.6 we also have in this case an exact triangle

$$
\underline{C}_{*}(F)_{c d h} \rightarrow \underline{C}_{*}(G)_{c d h} \rightarrow \underline{C}_{*}(H)_{c d h} \rightarrow \underline{C}_{*}(F)_{c d h}[1]
$$

and hence a long exact sequence of the corresponding hypercohomology groups.

We now prove localization and Mayer-Vietoris in our theory.

Theorem 5.11 Let $k$ be a field which admits resolution of singularities, let $X$ be a scheme of finite type over $k$, let $Y \subset X$ be a closed subscheme of $X$, and let $U_{1}, U_{2} \subset X$ be Zariski open subsets with $X=U_{1} \cup U_{2}$. Then there are canonical exact triangles (in the derived category of complexes of sheaves on $\left.(S m / k)_{Z a r}\right)$ of the form

$$
\begin{aligned}
\underline{C}_{*}\left(z_{\text {equi }}(Y, r)\right)_{Z a r} \rightarrow & \underline{C}_{*}\left(z_{\text {equi }}(X, r)\right)_{Z a r} \rightarrow \underline{C}_{*}\left(z_{\text {equi }}(X-Y, r)\right)_{Z a r} \rightarrow \\
& \rightarrow \underline{C}_{*}\left(z_{\text {equi }}(Y, r)\right)_{Z a r}[1]
\end{aligned}
$$

and

$$
\begin{gathered}
\underline{C}_{*}\left(z_{\text {equi }}(X, r)\right)_{Z a r} \rightarrow \underline{C}_{*}\left(z_{\text {equi }}\left(U_{1}, r\right)\right)_{Z a r} \oplus \underline{C}_{*}\left(z_{\text {equi }}\left(U_{2}, r\right)\right)_{Z a r} \rightarrow \\
\quad \rightarrow \underline{C}_{*}\left(z_{\text {equi }}\left(U_{1} \cap U_{2}, r\right)\right)_{Z a r} \rightarrow \underline{C}_{*}\left(z_{\text {equi }}(X, r)\right)_{Z a r}[1] .
\end{gathered}
$$


Proof: The sequences of presheaves

$$
\begin{gathered}
0 \rightarrow z_{\text {equi }}(Y, r) \rightarrow z_{\text {equi }}(X, r) \rightarrow z_{\text {equi }}(X-Y, r) \\
0 \rightarrow z_{\text {equi }}(X, r) \rightarrow z_{\text {equi }}\left(U_{1}, r\right) \oplus z_{\text {equi }}\left(U_{2}, r\right) \rightarrow z_{\text {equi }}\left(U_{1} \cap U_{2}, r\right)
\end{gathered}
$$

on $S m / k$ are exact for obvious reasons. Hence, by Lemma 2.7 and Theorem $5.5(2)$, it suffices to show that

$$
\begin{gathered}
\operatorname{coker}\left(i^{*}: z_{\text {equi }}(X, r) \rightarrow z_{\text {equi }}(X-Y, r)\right)_{\text {cdh }}=0 \\
\operatorname{coker}\left(j_{1}^{*}-j_{2}^{*}: z_{\text {equi }}\left(U_{1}, r\right) \oplus z_{\text {equi }}\left(U_{2}, r\right) \rightarrow z_{\text {equi }}\left(U_{1} \cap U_{2}, r\right)\right)_{c d h} .
\end{gathered}
$$

In view of definition of cdh-topology, to verify the first asserted vanishing it suffices to show for any smooth scheme $U$ over $k$ and any element $\mathcal{Z}$ in $z_{\text {equi }}(X-Y, r)(U)$ that there is a blow-up $p: U^{\prime} \rightarrow U$ such that $U^{\prime}$ is smooth and $\operatorname{cycl}(p)(\mathcal{Z})$ belongs to $z_{\text {equi }}(X, r)\left(U^{\prime}\right)$. We may clearly assume that $\mathcal{Z}=Z$ for a closed integral subscheme $Z$ of $(X-Y) \times U$. Let $\bar{Z}$ be the closure of $Z$ in $X \times U$. By the platification theorem [17], there is a blow-up $p: U^{\prime} \rightarrow U$ such that the proper transform $\tilde{Z}$ of $\bar{Z}$ with respect to $p$ is flat over $U^{\prime}$. Since $k$ admits resolution of singularities we may choose $U^{\prime}$ to be smooth. Then $\tilde{Z} \in z_{\text {equi }}(X, r)\left(U^{\prime}\right)$ and clearly its image in $z_{\text {equi }}(X-Y, r)\left(U^{\prime}\right)$ coincides with $\operatorname{cycl}(p)(Z)$.

The proof of the second asserted vanishing differs only in notation.

The following long exact sequence of bivariant cycle cohomology groups is an immediate corollary of Theorem 5.11 together with Lemma 3.6.

Corollary 5.12 Let $k$ be a field which admits resolution of singularities, let $X$ be a scheme of finite type over $k$, let $Y \subset X$ be a closed subscheme of $X$, and let $U_{1}, U_{2} \subset X$ be Zariski open subsets with $X=U_{1} \cup U_{2}$. Then for any scheme $U$ over $k$, there are canonical long exact sequences

$$
\begin{gathered}
\ldots \rightarrow A_{r, i}(U, Y) \rightarrow A_{r, i}(U, X) \rightarrow A_{r, i}(U, X-Y) \rightarrow A_{r, i-1}(U, Y) \rightarrow \ldots \\
\ldots \rightarrow A_{r, i}(Y, X) \rightarrow A_{r, i}\left(Y, U_{1}\right) \oplus A_{r, i}\left(Y, U_{2}\right) \rightarrow A_{r, i}\left(Y, U_{1} \cap U_{2}\right) \rightarrow \\
\rightarrow A_{r, i-1}(Y, X) \rightarrow \ldots
\end{gathered}
$$

The following theorem provides us with another class of long exact sequences in our theory. 
Theorem 5.13 Let $p: X^{\prime} \rightarrow X$ be a proper morphism of schemes of finite type over field $k$ which admits resolution of singularities and let and $Z \subset X$ be a closed subscheme of $X$ such that the morphism $p^{-1}(X-Z) \rightarrow X-Z$ is an isomorphism. Then there is a canonical exact triangle (in the derived category of complexes of sheaves on $\left.(S m / k)_{Z a r}\right)$ :

$$
\begin{gathered}
\underline{C}_{*}\left(z_{\text {equi }}\left(p^{-1}(Z), r\right)\right)_{Z a r} \rightarrow \underline{C}_{*}\left(z_{\text {equi }}(Z, r)\right)_{\text {Zar }} \oplus \underline{C}_{*}\left(z_{\text {equi }}\left(X^{\prime}, r\right)\right)_{Z a r} \rightarrow \\
\rightarrow \underline{C}_{*}\left(z_{\text {equi }}(X, r)\right)_{Z a r} \rightarrow \underline{C}_{*}\left(z_{\text {equi }}\left(p^{-1}(Z), r\right)\right)_{Z a r}[1] .
\end{gathered}
$$

Proof: The proof is exactly the same as for Theorem 5.11, except that we need the vanishing assertion

$$
\operatorname{coker}\left(z_{\text {equi }}(Z, r) \oplus z_{\text {equi }}\left(X^{\prime}, r\right) \rightarrow z_{\text {equi }}(X, r)\right)_{c d h}=0 .
$$

This is proved exactly as in the proof of the vanishing assertions of the proof of Theorem 5.11.

Corollary 5.14 Let $p: X^{\prime} \rightarrow X$ be a proper morphism of schemes of finite type over a field $k$ which admits resolution of singularities and let $Z \subset X$ be a closed subscheme of $X$ such that the morphism $p^{-1}(X-Z) \rightarrow X-Z$ is an isomorphism. Then for any scheme $U$ over $k$ there is a canonical long exact sequence of the form:

$$
\begin{aligned}
\ldots \rightarrow A_{r, i}\left(U, p^{-1}(Z)\right) & \rightarrow A_{r, i}(U, Z) \oplus A_{r, i}\left(U, X^{\prime}\right) \rightarrow A_{r, i}(U, X) \rightarrow \\
\rightarrow & A_{r, i-1}\left(U, p^{-1}(Z)\right) \rightarrow \ldots
\end{aligned}
$$

\section{Moving Lemma.}

In this section we return to the "naive" groups $\underline{h}_{i}\left(z_{\text {equi }}(X, r)\right)(U)$. We study them in the case of smooth varieties $X, U$ using the "moving lemma" technqiues developed in [9]. These techniques are summarized in Theorems 6.1 and 6.3 below. It is worthy of note that the results in this section apply to varieties over an arbitrary field $k$.

Let $X$ be an equidimensional smooth scheme of finite type over $k$. Let further $\mathcal{Z}=\sum n_{i} Z_{i}, \mathcal{W}=\sum m_{j} W_{j}$ be two effective cycles on $X$ of dimensions $r$ and $s$ respectively. We say that $\mathcal{Z}$ and $\mathcal{W}$ intersect properly if the schemes $Z_{i} \cap W_{j}$ are equidimensional of dimension $r+s-\operatorname{dim}(X)$.

We begin with a "presheafication" of the "Moving Lemma for Bounded Families" of cycles on projective space proved in [9]. 
Theorem 6.1 Let $k$ be a field and $m, r, s, d, e \geq 0$ be integers such that $r+s \geq m$. Then there are homomorphisms of abelian monoids

$$
\begin{aligned}
& H_{U}^{+}: z_{\text {equi }}^{\text {eff }}\left(\mathbf{P}^{m}, r\right)(U) \rightarrow z_{\text {equi }}^{\text {eff }}\left(\mathbf{P}^{m}, r\right)\left(U \times \mathbf{A}^{1}\right) \\
& H_{U}^{-}: z_{\text {equi }}^{\text {eff }}\left(\mathbf{P}^{m}, r\right)(U) \rightarrow z_{\text {equi }}^{\text {eff }}\left(\mathbf{P}^{m}, r\right)\left(U \times \mathbf{A}^{1}\right)
\end{aligned}
$$

defined for all smooth schemes $U$ over $k$ and satisfying the following conditions:

1. For any morphism $f: U^{\prime} \rightarrow U$ of smooth schemes over $k$, one has

$$
\begin{aligned}
& \theta_{U^{\prime}}^{+} \operatorname{cycl}(f)=\operatorname{cycl}\left(f \times I d_{\mathbf{A}^{1}}\right) H_{U}^{+} \\
& \theta_{U^{\prime}}^{-} \operatorname{cycl}(f)=\operatorname{cycl}\left(f \times I d_{\mathbf{A}^{1}}\right) H_{U}^{-}
\end{aligned}
$$

2. One has

$$
\begin{gathered}
\operatorname{cycl}_{i_{U}} \theta_{U}^{+}=(n+1) I d_{z_{\text {equi }}^{\text {eff }}\left(\mathbf{P}^{m}, r\right)(U)} \\
\operatorname{cycl}_{i_{U}} \theta_{U}^{-}=n I d_{z_{\text {equi }}^{\text {eff }}\left(\mathbf{P}^{m}, r\right)(U)}
\end{gathered}
$$

where $i_{U}$ is the closed embedding $I d_{U} \times\{0\}: U \rightarrow U \times \mathbf{A}^{1}$ and $n \geq 0$ is an integer.

3. For any geometric point

$$
\bar{x}: \operatorname{Spec}(\bar{k}) \rightarrow U \times\left(\mathbf{A}^{1}-\{0\}\right)
$$

any effective cycle $\mathcal{W}$ of degree $\leq e$ and dimension $s$ on $\mathbf{P}_{\bar{k}}^{m}$ and any cycle $\mathcal{Z} \in z_{\text {equi }}^{\text {eff }}\left(\mathbf{P}^{m}, r, \leq d\right)(U)$ the cycle $\mathcal{W}$ intersects properly in $\mathbf{P}_{\bar{k}}^{m}$ both $\operatorname{cycl}(\bar{x})\left(\theta_{U}^{+}(\mathcal{Z})\right)$ and $\operatorname{cycl}(\bar{x})\left(\theta_{U}^{-}(\mathcal{Z})\right)$.

Proof: As shown in $[9,3.1]$, there is a continuous algebraic map satisfying the analogues of the above properties (1), (2), (3) of the following form

$$
\tilde{\Theta}: \mathcal{C}_{r}\left(\mathbf{P}^{m}\right) \times V \rightarrow \mathcal{C}_{r}\left(\mathbf{P}^{m}\right)^{2},
$$

where $\mathcal{C}_{r}\left(\mathbf{P}^{m}\right)$ is the Chow monoid of effective $r$-cycles on $\mathbf{P}^{m}$ and $V \subset \mathbf{A}^{1}$ is a Zariski open neighborhood of $0 \in \mathbf{A}^{1}$. This map induces maps natural in $U$ :

$$
\mathcal{H}_{U, V}^{+}: \operatorname{Hom}\left(U, \mathcal{C}_{r}\left(\mathbf{P}^{m}\right)\right) \rightarrow \operatorname{Hom}\left(U \times V, \mathcal{C}_{r}\left(\mathbf{P}^{m}\right)\right)
$$




$$
\mathcal{H}_{U, V}^{-}: \operatorname{Hom}\left(U, \mathcal{C}_{r}\left(\mathbf{P}^{m}\right)\right) \rightarrow \operatorname{Hom}\left(U \times V, \mathcal{C}_{r}\left(\mathbf{P}^{m}\right)\right) .
$$

By Proposition 2.3, $z_{\text {equi }}^{\text {eff }}\left(\mathbf{P}^{m}, r\right)(U) \subset \operatorname{Hom}\left(U, \mathcal{C}_{r}\left(\mathbf{P}^{m}\right)\right)$ consists of those "graphs" of maps $f: U \rightarrow \mathcal{C}_{r}\left(\mathbf{P}^{m}\right)$ defined over $k$, so that the above maps restrict to maps

$$
\begin{aligned}
& H_{U, V}^{+}: z_{\text {equi }}^{e f f}\left(\mathbf{P}^{m}, r\right)(U) \rightarrow z_{\text {equi }}^{e f f}\left(\mathbf{P}^{m}, r\right)(U \times V) \\
& H_{U, V}^{-}: z_{\text {equi }}^{e f f}\left(\mathbf{P}^{m}, r\right)(U) \rightarrow z_{\text {equi }}^{e f f}\left(\mathbf{P}^{m}, r\right)(U \times V)
\end{aligned}
$$

satisfying (1), (2), (3) with $\mathbf{A}^{1}$ replaced by $V$.

To extend these maps to $U \times \mathbf{A}^{1}$, we let $u_{0}$ be a regular function on $\mathbf{A}^{1}$ whose divisor equals $W \equiv \mathbf{A}^{1}-V$ (as a reduced closed subscheme) and we let $w_{0}$ be another regular function on $\mathbf{A}^{1}$ whose divisor misses $W \cup\{0\}$ and whose degree is prime to $1+\operatorname{deg}\left(u_{0}\right)$. We set

$$
u=\frac{t^{\operatorname{deg}\left(u_{0}\right)+1}}{u_{0}}, w=\frac{t^{\operatorname{deg}\left(u_{0}\right)+\operatorname{deg}\left(w_{0}\right)+1}}{u_{0} w_{0}},
$$

where $t$ is the tautological regular function on $\mathbf{A}^{1}$. Let $\Gamma_{u}$ denote the graph of $u: V \rightarrow \mathbf{A}^{1}$, and let $\Gamma_{w}$ denote the graph of the rational map $w: W-->\mathbf{A}^{1}$. These graphs are finite over $\mathbf{A}^{1}$, in view of the fact that they equal the restrictions to $\mathbf{P}^{1} \times \mathbf{A}^{1}$ of graphs of morphisms from $\mathbf{P}^{1}$ to itself.

Let $m, n$ be chosen so that $m \operatorname{deg}(u)+n \operatorname{deg}(w)=1$ and set $\Gamma=m \Gamma_{u}+n \Gamma_{w}$. Then, $\Gamma \in C_{\text {equi }}\left(V \times \mathbf{A}^{1} / \mathbf{A}^{1}, 0\right)$, a smooth relative curve over $\mathbf{A}^{1}$. We consider the composition

$$
\begin{gathered}
\Psi=\phi(\Gamma) \circ \pi^{*}: z_{\text {equi }}^{e f f}(Y, r)\left(\mathbf{P}^{m}, r\right)(U \times V) \rightarrow z_{\text {equi }}^{e f f}(Y, r)\left(\mathbf{P}^{m}, r\right)\left(U \times V \times \mathbf{A}^{1}\right) \\
\rightarrow z_{\text {equi }}^{e f f}(Y, r)\left(\mathbf{P}^{m}, r\right)\left(U \times \mathbf{A}^{1}\right),
\end{gathered}
$$

the composition of flat pull-back followed by the "transfer" with respect to $\Gamma$ for the pre-theoretical structure of $z_{\text {equi }}\left(\mathbf{P}^{m}, r\right)$. The latter map is described more explicitly as follows. For each

$$
\mathcal{Z} \in z_{\text {equi }}^{e f f}(Y, r)\left(\mathbf{P}^{m}, r\right)\left(U \times V \times \mathbf{A}^{1}\right)
$$

and each generic point $\gamma$ of an irreducible component $G_{\gamma}$ of $U \times \Gamma$ in $U \times$ $V \times \mathbf{A}^{1}$, we consider the push-forward to $\mathbf{P}^{m} \times \mathbf{A}^{1}$ of the closure in $\mathbf{P}^{m} \times G_{\gamma}$ of the specializaton $\mathcal{Z}_{\gamma}$ on $\mathbf{P}^{m} \times \operatorname{Spec}(k(\gamma))$. Then,

$$
\phi(\Gamma)(\mathcal{Z})=\sum_{\gamma}\left(1 \times p_{\gamma}\right)_{*}\left(\overline{\mathcal{Z}}_{\gamma}\right),
$$


where $p_{\gamma}: G_{\gamma} \rightarrow U \times \mathbf{A}^{1}$.

To verify that

$$
H_{U}^{+} \equiv \Psi \circ \mathcal{H}_{U, V}^{+}, H_{U}^{-} \equiv \Psi \circ \mathcal{H}_{U, V}^{-}
$$

satisfy (1), (2), and (3), we consider for each $x \in U$ the following diagram

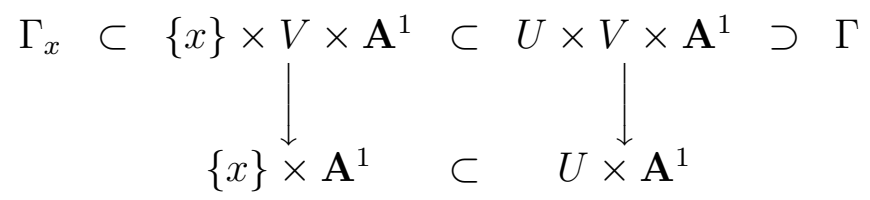

By property (3) of the pretheoretical structure for $z_{\text {equi }}\left(\mathbf{P}^{m}, r\right)$, we obtain the commutative square

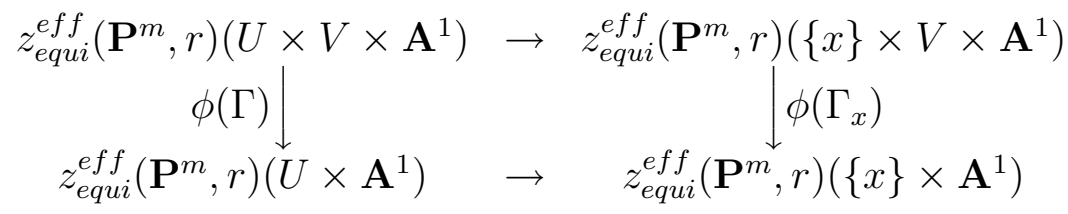

Property (1) is immediate. Since the restriction $\Gamma_{0}$ of $\Gamma$ to $\{o\} \times \mathbf{A}^{1}$ is the graph of the embedding $\{0\} \subset \mathbf{A}^{1}$, we conclude that $\phi\left(\Gamma_{x}\right) \circ \pi^{*}=i d$, property (2) follows. Finally, property (3) follows easily from commutative square $\left(^{*}\right)$ and the identification of $\Gamma_{x}$ for $x \neq 0$ as the sum of graphs of embeddings of $\{x\}$ into $\mathbf{A}^{1}-\{0\}$.

This theorem provides us with a method to move families of cycles on projective space. To move families of cycles on more general projective varieties, one uses a version of classical projective cones technique (see [18]) as developed in [9].

Let $X \subset \mathbf{P}^{n}$ be a smooth equidimensional projective variety of dimension $m$ over $k$. For a positive integer $D$, we consider the projective space $\mathbf{P}\left(H^{0}(\mathcal{O}(D))^{m+1}\right)$ which parametrize projections $\mathbf{P}^{n} \rightarrow \mathbf{P}^{m}$ given by families $\underline{F}=\left(F_{0}, \ldots, F_{m}\right)$ of homogeneous polynomials of degree $D$. Let further $\mathcal{U}_{X, D}$ be the open subscheme in this projective space which consists of families $\underline{F}$ such that the corresponding rational map $\pi_{E}: \mathbf{P}^{n} \rightarrow \mathbf{P}^{m}$ restricts to a finite flat morphism $p_{\underline{F}}: X \rightarrow \mathbf{P}^{m}$. Note that this open subscheme is always nonempty. 
For an effective cycle $\mathcal{Z}$ on $X$ and a point $\underline{F}$ of $\mathcal{U}_{X, D}$ denote by $R_{\underline{F}}(\mathcal{Z})$ the effective cycle $p_{\underline{F}}^{*}\left(p_{\underline{F}}\right)_{*}(\mathcal{Z})-\mathcal{Z}$. Since this construction only uses flat pull-backs and proper push-forwards of cycles it has a relative analog. More precisely, Proposition 2.1 imply that any point $\underline{F}$ of $\mathcal{U}_{X, D}$ defines an endomorphism of presheaves

$$
R_{\underline{F}}: z_{\text {equi }}^{e f f}(X, r) \rightarrow z_{\text {equi }}^{e f f}(X, r) .
$$

For a point $\underline{F}$ in $\mathcal{U}_{X, D}$ denote by $\operatorname{Ram}_{\underline{F}}$ the closed subset in $X$ which consists of ramification points of the projection $p_{\underline{F}}: X \rightarrow \mathbf{P}^{m}$. The key result of the projective cones technique is the following proposition.

Proposition 6.2 Let $k$ be an field, $X \subset \mathbf{P}^{n}$ be a smooth equidimensional projective scheme of dimension $m$ and $d, e, r, s \geq 0$ be integers such that $r+s \geq m$. Then there exists $D>0$ and a nonempty open subscheme $\mathcal{V}=$ $\mathcal{V}_{d, e, r, s}$ in $\mathcal{U}_{X, D}$ such that for any point $\underline{F}$ in $\mathcal{V}$ and any pair of effective cycles $\mathcal{Z}, \mathcal{W}$ on $X$ of degrees $d, e$ and dimensions $r, s$ respectively all components of dimension $>r+s-m$ in $\operatorname{Supp}(\mathcal{W}) \cap \operatorname{Supp}\left(R_{\underline{F}}(\mathcal{Z})\right)$ belong to $\operatorname{Supp}(\mathcal{W}) \cap$ $\operatorname{Supp}(\mathcal{Z}) \cap \operatorname{Ram}_{\underline{F}}$.

Proof: See $[9,1.7]$.

We apply Proposition 6.2 several times to obtain a sequence $\underline{F}_{0}, \ldots, \underline{F}_{m}$ of points in $\mathcal{U}_{X, D_{i}}$ for some $D_{0}, \ldots, D_{m}>0$ such that for any effective cycle $\mathcal{Z}$ of dimension $r$ and degree $d$ the (effective) cycle $R_{\underline{F}_{m}} \ldots R_{\underline{E}_{0}}(\mathcal{Z})$ intersects properly all effective cycles of dimesnion $s$ and degree $e$ (provided $r+s \geq$ $m$ ). Since the cycle $R_{\underline{F}_{m}} \ldots R_{\underline{E}_{0}}(\mathcal{Z})$ differs from the cycle $\mathcal{Z}$ by a linear combination of pull-backs of cycles on $\mathbf{P}^{m}$ we may further apply Theorem 6.1 to obtain a "move" of any relative cycle which will intersect given relative cycles properly. The precise formulation of the corresponding "moving lemma for families of cycles" is given by the following theorem.

Theorem 6.3 Let $k$ be a field, $X \subset \mathbf{P}^{n}$ be a smooth projective equidimensional scheme of dimension $m$ over $k$ and $d, e, r, s \geq 0$ be integers such that $r+s \geq m$. Then there are homomorphisms of abelian monoids

$$
\begin{aligned}
& H_{U}^{+}: z_{\text {equi }}^{\text {eff }}(X, r)(U) \rightarrow z_{\text {equi }}^{\text {eff }}(X, r)\left(U \times \mathbf{A}^{1}\right) \\
& H_{U}^{-}: z_{\text {equi }}^{\text {eff }}(X, r)(U) \rightarrow z_{\text {equi }}^{\text {eff }}(X, r)\left(U \times \mathbf{A}^{1}\right)
\end{aligned}
$$


defined for all smooth schemes $U$ over $k$ and satisfying the following conditions:

1. For any morphism $f: U^{\prime} \rightarrow U$ of smooth schemes over $k$ one has

$$
\begin{aligned}
& H_{U^{\prime}}^{+} \operatorname{cycl}(f)=\operatorname{cycl}\left(f \times I d_{\mathbf{A}^{1}}\right) H_{U}^{+} \\
& H_{U^{\prime}}^{-} \operatorname{cycl}(f)=\operatorname{cycl}\left(f \times I d_{\mathbf{A}^{1}}\right) H_{U}^{-}
\end{aligned}
$$

2. Let $\mathcal{W}$ be an effective cycle of degree $\leq e$ and dimension $s$ on $X_{\bar{k}}$ and $\mathcal{Z}$ be an element of $z_{\text {equi }}^{\text {eff }}(X, r, \leq d)(U)$. Then for any geometric point $\bar{x}: \bar{k} \rightarrow U \times\{0\} \subset U \times \mathbf{A}^{1}$ of $U$ one has:

(a) The components of the closed subschemes

$$
\begin{aligned}
& \operatorname{Supp}\left(\operatorname{cycl}(\bar{x})\left(H_{U}^{+}(\mathcal{Z})\right)\right) \cap \operatorname{Supp}(\mathcal{W}) \\
& \operatorname{Supp}\left(\operatorname{cycl}(\bar{x})\left(H_{U}^{-}(\mathcal{Z})\right)\right) \cap \operatorname{Supp}(\mathcal{W})
\end{aligned}
$$

of dimension $>r+s-m$ belong to $\operatorname{Supp}(\operatorname{cycl}(\bar{x})(\mathcal{Z})) \cap \operatorname{Supp}(\mathcal{W})$.

(b)

$$
\operatorname{cycl}(\bar{x})\left(H_{U}^{+}(\mathcal{Z})\right)=\operatorname{cycl}(\bar{x})\left(H_{U}^{-}(\mathcal{Z})\right)+\mathcal{Z} .
$$

3. For any geometric point $\bar{x}: \operatorname{Spec}(\bar{k}) \rightarrow U \times\left(\mathbf{A}^{1}-\{0\}\right)$, any effective cycle $\mathcal{W}$ of degree $\leq e$ and dimension $s$ on $X_{\bar{k}}$ and any cycle $\mathcal{Z} \in z_{\text {equi }}^{\text {eff }}(X, r, \leq d)(U)$ the cycles $\mathcal{W}$ and $\operatorname{cycl}(\bar{x})\left(H_{U}^{+}(\mathcal{Z})\right)$ (resp. $\left.\operatorname{cycl}(\bar{x})\left(H_{U}^{-}(\mathcal{Z})\right)\right)$ on $X_{\bar{k}}$ intersect properly.

Proof: See $[9,3.2]$.

Let $T=X \times Y$ be a product of projective, smooth schemes over $k$ and let $e \geq 0$ be such that $\{x\} \times Y \subset T$ has degree $\leq e$ for all points $x \in X$. Then Theorem 6.3 asserts that $\mathcal{Z} \in z_{\text {equi }}^{e f f}(T, r)(U)$ can be moved to $\mathcal{Z}^{\prime}$ on $T \times U$ with the property that $\operatorname{cycl}(\bar{u})\left(\mathcal{Z}^{\prime}\right)$ meets $\{x\} \times Y \times \operatorname{Spec}(\bar{k})$ properly for all geometric points $\bar{u}: \operatorname{Spec}(\bar{k}) \rightarrow U$, all points $x \in X$. The following proposition enables us to conclude that such a $\mathcal{Z}^{\prime}$ lies in the image of $z_{\text {equi }}^{e f f}(X, r)(U \times Y)$. 
Proposition 6.4 Let $X$ be a smooth scheme of pure dimension $n$ over a field $k$, let $Y$ be a projective scheme over $k$, and let $U$ be a smooth scheme over $k$. Then $\mathcal{Z} \in z_{\text {equi }}^{e f f}(X \times Y, r+n)(U)$ lies in the image of

$$
\mathcal{D}^{\text {eff }}: z_{\text {equi }}(Y, r)^{\text {eff }}(X \times U) \rightarrow z_{\text {equi }}(X \times Y, r+n)^{\text {eff }}(U)
$$

if and only if for every geometric point $\bar{u}: \operatorname{Spec}(\bar{k}) \rightarrow U$ the cycle cycl $(\bar{u})(\mathcal{Z})$ belongs to the image of the homomorphism

$$
\mathcal{D}^{\text {eff }}: z_{\text {equi }}(Y, r)^{\text {eff }}(X \times \operatorname{Spec}(\bar{k})) \rightarrow z_{\text {equi }}(X \times Y, r+n)^{\text {eff }}(\operatorname{Spec}(\bar{k})) .
$$

Proof: We may assume that $\mathcal{Z}$ is a closed integral subscheme of $X \times Y \times U$ satisfying the condition that for every geometric point $\bar{u}: \operatorname{Spec}(\bar{k}) \rightarrow U$ the subscheme $Z_{\bar{u}} \subset X \times Y \times \operatorname{Spec}(\bar{k})$ is equidimensional over $X \times \operatorname{Spec}(\bar{k})$. The generic fibre of the projection $\mathcal{Z} \rightarrow X \times U$ defines a rational map $\phi_{\mathcal{Z}}: X \times U--->\mathcal{C}_{r}(Y)$. It suffices to prove that the graph $\Gamma\left(\phi_{\mathcal{Z}}\right) \subset$ $X \times U \times \mathcal{C}_{r}(Y)$ projects bijectively onto $X \times U$, since the resulting continuous algebraic map is a morphism by the normality of $X \times U$.

To prove the required bijectivity, it suffices to show for any specialization $\eta \searrow \bar{x} \times \bar{u}$ in $X \times U$ and any point $\mathcal{Z}_{\eta} \in \Gamma\left(\phi_{\mathcal{Z}}\right)$ that the only specialization of $\mathcal{Z}_{\eta}$ extending $\eta \searrow \bar{x} \times \bar{u}$ is $\mathcal{Z}_{\eta} \searrow f_{\bar{u}}(\bar{x})$, where $f_{\bar{u}}: X \times \operatorname{Spec}(\bar{k}) \rightarrow$ $\mathcal{C}_{r}(Y)$ is associated to $\operatorname{cycl}(\bar{u})(\mathcal{Z}) \in z_{\text {equi }}(Y, r)^{\text {eff }}(X \times \operatorname{Spec}(\bar{k}))$. This is a consequence of the observation that if $\mathcal{Z}_{C} \subset C \times Y$ is a cycle assoicated to a map $f: C \rightarrow \mathcal{C}_{r}(Y)$ for some smooth curve $C$, then for any geometric point $\bar{c}: \operatorname{Spec}(\bar{k}) \rightarrow C$ the Chow point of the cycle $\mathcal{Z}_{\bar{x}} \subset Y \times \operatorname{Spec}(\bar{k})$ equals $f(\bar{c})$.

The main reason we have worked explicitly with differences of effective cycles instead of all cycles is the fact that Proposition 6.4 becomes false without effectivity assumption on $\mathcal{Z}$ as shown in the following example.

Example 6.5 Let $V=\mathbf{A}^{1}, U=X=\mathbf{P}^{1}$. Consider the cycle $W=W_{+}-W_{-}$ on $V \times U \times X$ where $W_{+}$(resp. $W_{-}$) is the graph of the rational map $U \times V \rightarrow X$ of the form $x / y$ (resp. 2x/y). Then both $W_{+}$and $W_{-}$are relative cycles over $V$. Moreover the specialization of $W$ (but not of $W_{+}$or $W_{-}$!) to any point $v$ of $V$ is a relative cycle on $(U \times X)_{v}$ over $U_{v}$, but $W$ is not relative over $U \times V$.

In order to apply Theorem 6.3 in the next section to prove that the "duality map" is a quasi-isomorphism, we shall require the following simple result. 
Lemma 6.6 Let $k$ be a field, $F$ be a presheaf of abelian groups on the category $S m / k$ and $G \subset F$ be a subpresheaf (of abelian groups) of F. Let further

$$
F_{0} \subset F_{1} \subset \ldots \subset F_{n} \subset \ldots
$$

be an increasing sequence of subpresheaves of sets in $F$ such that $F=\cup_{d \geq 0} F_{d}$.

Assume that for any $d \geq 0$ there exist a family of homomorphisms

$$
H_{U}: F(U) \rightarrow F\left(U \times \mathbf{A}^{1}\right)
$$

given for all smooth schemes $U$ over $k$ and satisfying the following conditions:

1. For any morphism $f: U^{\prime} \rightarrow U$ one has $H_{U^{\prime}} F(f)=F\left(f \times I d_{\mathbf{A}^{1}}\right) H_{U^{\prime}}$.

2. For any smooth scheme $U$ one has

$$
\begin{gathered}
F\left(i_{1}\right) H_{U}\left(F_{d}(U)\right) \subset G(U) \\
F\left(i_{0}\right) H_{U}=I d_{F(U)}
\end{gathered}
$$

where $i_{0}, i_{1}$ are the closed embeddings $I d_{U} \times\{0\}$ and $I d_{U} \times\{1\}$ respectively.

3. For any smooth scheme $U$ one has

$$
H_{U}\left(G \cap F_{d}\right) \subset G
$$

Then the morphism of complexes of presheaves $\underline{C}_{*}(G) \rightarrow \underline{C}_{*}(F)$ is a quasi-isomorphism.

Proof: As in the proof of Lemma 4.1, the natural (with respect to $U$ ) homomorphism $H_{U}$ determines a natural chain homotopy

$$
s_{*}: \underline{C}_{*}(F)(U) \rightarrow \underline{C}_{*+1}(F)(U)
$$

whose restriction to $\underline{C}(G)_{*}(U)$ lies in $\underline{C}_{*+1}(G)(U)$ and which relates the identity to a map $h_{U}=F\left(i_{1}\right) \circ H_{U}$ satisfying $h_{U}\left(\underline{C}\left(F_{d}\right)_{*}(U)\right) \subset \underline{C}(G)_{*}(U)$. Since $F=\cup_{d \geq 0} F_{d}$, this easily implies that $\underline{C}_{*}(G)(U) \rightarrow \underline{C}_{*}(F)(U)$ is a quasiisomorphism. 


\section{Duality.}

In this section, we prove duality theorems relating $z_{\text {equi }}(U, X, r)$ to $z_{\text {equi }}(X \times$ $U, r+\operatorname{dim}(U))$ for a smooth scheme $U$. The proofs of these theorems use techniques which were originally developed for the duality theorems of [7]. In the next section, we shall apply duality to conclude the basic properties of bivariant cycle cohomology groups $A_{r, i}(Y, X)$ for all schemes of finite type $Y, X$ over a field $k$ which admit resolution of singularities.

We begin with the following duality theorem for projective, smooth varieties over an arbitrary field $k$.

Theorem 7.1 Let $X, Y$ be smooth projective equidimensional schemes over a field $k$. Then the embedding of presheaves

$$
\mathcal{D}: z_{\text {equi }}(Y, X, r) \rightarrow z_{\text {equi }}(X \times Y, r+\operatorname{dim}(Y))
$$

induces isomorphisms

$$
\underline{h}_{i}\left(z_{\text {equi }}(Y, X, r)\right) \rightarrow \underline{h}_{i}\left(z_{\text {equi }}(X \times Y, r+\operatorname{dim}(Y))\right)
$$

for all $i \in \mathbf{Z}$.

Proof: We apply Theorem 6.3 with $X$ replaced by $X \times Y \subset \mathbf{P}^{N}$ and $e$ the maximum of the degrees of $x \times Y \subset X \times Y$. Lemma 6.6 enables us to obtain from Theorem 6.3 a quasi-isomorphism of complexes of presheaves. We interpret this as the required quasi-isomorphism

$$
\underline{C}_{*}\left(z_{\text {equi }}(X, Y, r) \rightarrow \underline{C}_{*}(x(X \times Y, r)\right.
$$

by applying Proposition 6.4

We now proceed to remove the hypotheses that $X, Y$ be projective and smooth but add the hypothesis that $k$ admit resolution of singularities. In so doing, we shall obtain a quasi-isomorphism of chain complexes obtained by evaluating the appropriate complexes of presheaves at $\operatorname{Spec}(\bar{k})$. The key reason why we do not conclude a quasi-isomorphism of presheaves is that the conclusion of Theorem 5.5(2) concerns the associated sheaf of a presheaf.

For the remainder of this section, let $k$ a field which admits resolution of singularities, let $U$ be a smooth scheme over $k$ of pure dimension $n$, let 
$i_{U}: U \subset \bar{U}$ be a smooth compactification, let $X$ be a scheme of finite type over $k$, and let $i_{X}: X \subset \bar{x}$ be an embedding of $X$ in a proper scheme of finite type over $k$ (see [14]).

For any proper scheme $q: \bar{Y} \rightarrow \bar{X}$ of finite type over $\bar{X}$, consider the morphism of presheaves of abelian monoids

$$
\alpha_{\bar{Y}}^{e f f}: z_{\text {equi }}^{e f f}(\bar{U} \times \bar{Y}, n+r) \rightarrow z_{\text {equi }}^{\text {eff }}(U \times X, n+r)
$$

which is the composition of proper push-forward morphism

$$
\left(I d_{\bar{U}} \times q\right)_{*}: z_{\text {equi }}^{e f f}(\bar{U} \times \bar{Y}, n+r) \rightarrow z_{\text {equi }}^{e f f}(\bar{U} \times \bar{X}, n+r)
$$

with the flat pull-back

$$
\left(i_{U} \times i_{X}\right)^{*}: z_{\text {equi }}^{e f f}(\bar{U} \times \bar{X}, n+r) \rightarrow z_{\text {equi }}^{e f f}(U \times X, n+r) .
$$

Let $\alpha_{\bar{Y}}$ be the corresponding morphism of presheaves of abelian groups.

We denote by $\Phi_{\bar{Y}}$ the subpresheaf of abelian groups in $z_{\text {equi }}(\bar{U} \times \bar{Y}, n+r)$ generated by subpresheaf of abelian monoids $\left(\alpha_{\bar{Y}}^{\text {eff }}\right)^{-1}\left(z_{\text {equi }}^{\text {eff }}(U, X, r)\right)$ (where we identify $z_{\text {equi }}^{\text {eff }}(U, X, r)$ with its image in $\left.z_{\text {equi }}^{\text {eff }}(U \times X, r+n)\right)$. Thus, $\Phi_{\bar{Y}}$ fits in the following commutative square

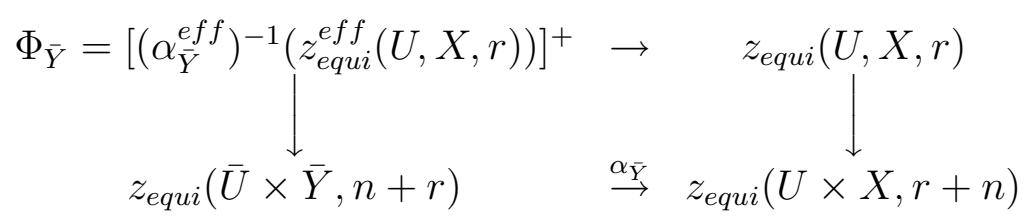

where $[-]^{+}$denotes the abelian group associated with the abelian monoid - .

We should be wary that for general $q: \bar{Y} \rightarrow \bar{X}$,

$$
\Phi_{\bar{Y}} \neq\left(\alpha_{\bar{Y}}\right)^{-1}\left(z_{\text {equi }}(U, X, r)\right) ;
$$

for example, distinct effective cycles on $\bar{U} \times \bar{Y}$ might map to the same, nonequidimensional cycle on $\bar{U} \times \bar{X})$. Of course, $\Phi_{\bar{X}} \neq\left(\alpha_{\bar{X}}\right)^{-1}\left(z_{\text {equi }}(U, X, r)\right.$.

We require the following lemma, analgous to but considerably more elementary than Proposition 6.4.

Lemma 7.2 Let $V$ be a smooth scheme over $k, q: \bar{Y} \rightarrow \bar{X}$ be a smooth projective scheme over $\bar{X}$ and $\mathcal{Z}=\sum n_{i} Z_{i}\left(n_{i} \neq 0\right)$ be an element of $z_{\text {equi }}(\bar{U} \times$ 
$\bar{Y}, n+r)(V)$. It belongs to $\Phi_{\bar{Y}}$ if and only if for any geometric point $\bar{x}$ : $\operatorname{Spec}(\bar{k}) \rightarrow V \times U$ of $V \times U$ and any $i$ we have

$$
\operatorname{dim}\left(q_{\bar{k}}\left(Z_{i} \times_{V \times U} \operatorname{Spec}(\bar{k})\right) \cap X_{\bar{k}}\right) \leq r
$$

where

$$
\begin{aligned}
q_{\bar{k}} & =q \times_{\operatorname{Spec}(k)} \operatorname{Spec}(\bar{k}) \\
X_{\bar{k}} & =X \times_{\operatorname{Spec}(k)} \operatorname{Spec}(\bar{k}) .
\end{aligned}
$$

Proof: Note first that due to our definition of $\Phi_{\bar{Y}}$ we may assume that $\mathcal{Z}=Z$ for a closed integral subscheme $Z$ in $V \times \bar{U} \times \bar{Y}$ which is equidimensional of relative dimension $r+n$ over $V$. Obviously $Z \in \Phi_{\bar{Y}}(V)$ if and only if

$$
\left(I d_{V} \times I d_{\bar{U}} \times q\right)(Z) \cap V \times U \times X
$$

is equidimensional of relative dimension $r$ over $V \times U$ or is of dimension everywhere less then $r$ over $U \times V$ (the last case corresponds to $\alpha_{\bar{Y}}(\mathcal{Z})=0$ ). Equivalently, this means that for any geometric point $\bar{x}$ of $V \times U$ we have

$$
\operatorname{dim}\left(\left(I d_{V} \times I d_{\bar{U}} \times q\right)(Z) \times_{V \times U} \operatorname{Spec}(\bar{k}) \cap X_{\bar{k}}\right) \leq r .
$$

Our statement follows now from the obvious equality:

$$
\left(I d_{V} \times I d_{\bar{U}} \times q\right)(Z) \times_{V \times U} \operatorname{Spec}(\bar{k})=q_{\bar{k}}\left(Z \times_{V \times U} \operatorname{Spec}(\bar{k})\right) .
$$

The following somewhat technical proposition generalizes Theorem 7.1 in the following sense: if $X$ is projective but not necessarily smooth and if $U$ is both projective and smooth, then Proposition 7.3 immediately implies the quasi-isomorphism of chain complexes

$$
\underline{C}_{*}\left(z_{\text {equi }}(U, X, r)\right)(\operatorname{Spec}(k)) \rightarrow \underline{C}_{*}\left(z_{\text {equi }}(X \times U, r+n)\right)(\operatorname{Spec}(k)) .
$$

Proposition 7.3 Let $q: \bar{Y} \rightarrow \bar{X}$ be a proper scheme over $\bar{X}$. Then for any smooth scheme $U$ of pure dimension $n$ and any $i \in \mathbf{Z}$, the morphisms

$$
\underline{h}_{i}\left(\Phi_{\bar{Y}}\right)(\operatorname{Spec}(k)) \rightarrow \underline{h}_{i}\left(z_{\text {equi }}(\bar{U} \times \bar{Y}, r+n)\right)(\operatorname{Spec}(k))
$$

are isomorphisms. 
Proof: We first assume that $\bar{Y}$ is smooth; then we may clearly also assume that $\bar{Y}$ is connected (and thus equidimensional). Choose a projective embed$\operatorname{ding} \bar{U} \times \bar{Y} \subset \mathbf{P}^{N}$. Let $e$ be the degree of closed subschemes $(\bar{Y})_{\bar{u}}, \bar{u} \in U(\bar{k})$ of $\mathbf{P}_{\bar{k}}^{N}$ and let $d \geq 0$ be an integer. Applying Theorem 6.3 to the scheme $\bar{U} \times \bar{Y}$ (with numbers being $d, e, r+n, \operatorname{dim}(\bar{Y})$ ) we get a family of natural homomorphisms

$$
H_{V}=H_{V}^{+}-H_{V}^{-}: z_{\text {equi }}(\bar{U} \times \bar{Y}, n+r)(V) \rightarrow z_{\text {equi }}(\bar{U} \times \bar{Y}, n+r)\left(V \times \mathbf{A}^{1}\right)
$$

given for all smooth schemes $V$ over $k$. It is sufficient to show now that they satisfy conditions of Proposition 6.6 (for $F=z_{\text {equi }}(\bar{U} \times \bar{Y}, n+r), F_{d}=$ $z_{\leq d}(\bar{U} \times \bar{Y}, n+r)$ and $\left.G=\Phi_{\bar{Y}}\right)$. Property (1) follows from Theorem 6.3(1). Property (2) follows from our choice of $e$, Lemma 7.2 and Theorem6.3(2b, $3)$. Finally the property (3) follows from Lemma 7.2 and Theorem 6.3(2a,3).

We now consider the general case in which $q: \bar{Y} \rightarrow \bar{X}$ is a proper scheme over $\bar{X}$ of dimension $\leq \operatorname{dim}(X)$. By the above proof for smooth $\bar{Y}$, we may assume that our quasi-isomorphism is proven for all smooth projective schemes as well as for all schemes of dimension $<\operatorname{dim}(\bar{Y})$.

Since $k$ admits resolution of singularities there is a proper surjective morphism $p: \bar{Y}^{\prime} \rightarrow \bar{Y}$ such that $\bar{Y}^{\prime}$ is smooth and projective. and there exits a closed subscheme $j: \bar{Z} \rightarrow \bar{Y}$ in $\bar{Y}$ such that $p^{-1}(\bar{Y}-\bar{Z}) \rightarrow \bar{Y}-\bar{Z}$ is an isomorphism and $\operatorname{dim}(\bar{Z})<\operatorname{dim}(\bar{Y})$. Consider the following diagram of presheaves (we write $z_{\text {equi }}(-)$ instead of $z_{\text {equi }}(-, r+n)$ ):

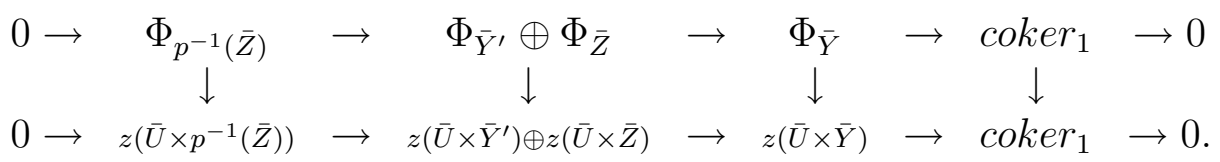

Since $\operatorname{dim}\left(p^{-1}(\bar{Z})\right)<\operatorname{dim}(\bar{Y})$, the induction assumption together with the isomorphism for the smooth, projective $\bar{Y}$ imply that the first two vertical arrows induce isomorphisms on the corresponding groups $\underline{h}_{i}(-)(\operatorname{Spec}(k))$. One can verify easily that both horizontal sequences are exact and the last vertical arrow is a monomorphism. Proposition 5.8 implies that all horizontal and vertical arrows are morphisms of pretheories. Applying Lemma 3.6 and Theorem 5.5(2) to coker $_{j}$ we see that it is sufficient to show that $\left(\text { coker }_{2}\right)_{c d h}=$ 0 . This follows exactly as in the proof of Theorem 5.11 (cf. Theorem 5.13.

We now can easily prove our main duality theorem. 
Theorem 7.4 Let $k$ be a field which admits resolution of singularities, $U$ be a smooth quasi-projective equidimensional scheme of dimension $n$ over $k$ and $X$ be a scheme of finite type over $k$. Then for any $r \geq 0$ the embedding

$$
\mathcal{D}: z_{\text {equi }}(U, X, r) \rightarrow z_{\text {equi }}(X \times U, r+n)
$$

induce quasi-isomorphisms of complexes of abelian groups

$$
\underline{C}_{*}\left(z_{\text {equi }}(U, X, r)\right)(\operatorname{Spec}(k)) \rightarrow \underline{C}_{*}\left(z_{\text {equi }}(X \times U, r+n)\right)(\operatorname{Spec}(k)) .
$$

Proof: We have the following diagram of morphisms of presheaves

$$
\begin{aligned}
& 0 \rightarrow \text { ker }^{\prime} \quad \rightarrow \quad \Phi_{\bar{X}} \quad \rightarrow \quad z_{\text {equi }}(U, X) \rightarrow \text { coker }_{1} \rightarrow 0 \\
& a \downarrow \quad b \downarrow \quad \mathcal{D} \downarrow \quad c \downarrow \\
& 0 \rightarrow \operatorname{ker}\left(\alpha_{\bar{X}}\right) \rightarrow z_{\text {equi }}(\bar{U} \times \bar{X}) \rightarrow z_{\text {equi }}(U \times X) \rightarrow \text { coker }_{2} \rightarrow 0 .
\end{aligned}
$$

(where

$$
\begin{gathered}
z_{\text {equi }}(U, X)=z_{\text {equi }}(U, X, r) \\
z_{\text {equi }}(\bar{U} \times \bar{X})=z_{\text {equi }}(\bar{U} \times \bar{X}, r+n) \\
\left.z_{\text {equi }}(U \times X)=z_{\text {equi }}(U \times X, r+n)\right)
\end{gathered}
$$

Since $\Phi_{\bar{X}}=\left(\alpha_{\bar{X}}\right)^{-1}\left(z_{\text {equi }}(U, X, r)\right)$, the morphism $a$ is an isomorphism and all other vertical morphisms are monomorphisms. Moreover, Proposition 7.3 asserts that $b(\operatorname{Spec}(\bar{k}))$ is a quasi-isomorphism. Thus, it suffices to show that

$$
\underline{h}_{i}\left(\operatorname{coker}_{j}\right)(\operatorname{Spec}(k))=0
$$

for $j=1,2$ and all $i \in \mathbf{Z}$. By Proposition 5.8(2) (resp. 5.8(3)) the morphism $\alpha($ resp. $\mathcal{D})$ is a morphism of pretheories. In particular coker ${ }_{j}$ are pretheories. In view of Theorem 5.5(2) it is sufficient to show that $\left(\text { coker }_{j}\right)_{c d h}=0$. Since coker $_{1} \subset$ coker $_{2}$ Lemma 3.6 implies we have only to consider the case of coker $_{2}$ whose vanishing is given in the proof of Theorem 5.11.

\section{Properties and pairings.}

We can prove now the comparison theorem for "naive" $\left(\underline{h}_{i}\left(z_{\text {equi }}(X, r)(U)\right)\right.$ and "fancy" $\left(A_{r, i}(U, X)\right)$ definitions of bivariant cycle cohomology discussed in Section 4. 
Theorem 8.1 Let $k$ be a field which admits a resolution of singularities, let $X$ be a scheme of finite type over $k$, and $U$ be a smooth quasi-projective scheme over $k$. Then the natural homomorphisms of abelian groups

$$
\underline{h}_{i}\left(z_{\text {equi }}(X, r)\right)(U) \rightarrow A_{r, i}(U, X)
$$

are isomorphisms for all $i \in \mathbf{Z}$.

Proof: For any smooth scheme $V$ of pure dimension $n$ and Zariski open subsets $V_{1}, V_{2} \subset V$ with $V=V_{1} \cup V_{2}$, we have the following exact sequences of complexes

$$
\begin{aligned}
0 \rightarrow \underline{C}_{*}\left(z_{\text {equi }}(X, r)\right)(V) & \rightarrow \underline{C}_{*}\left(z_{\text {equi }}(X, r)\right)\left(V_{1}\right) \oplus \underline{C}_{*}\left(z_{\text {equi }}(X, r)\right)\left(V_{2}\right) \rightarrow \\
& \rightarrow \underline{C}_{*}\left(z_{\text {equi }}(X, r)\right)\left(V_{3}\right)
\end{aligned}
$$

where $V_{3}=V_{1} \cap V_{2}$. By Theorem 7.4, this sequence is quasi-isomorphic in the derived category to the distinguished triangle obtained by evaluating the following distinguished triangle provided by Theorem 5.11

$$
\begin{gathered}
\underline{C}_{*}\left(z_{\text {equi }}(X \times V, r+n) \rightarrow \underline{C}_{*}\left(z_{\text {equi }}\left(X \times V_{1}, r+n\right)\right) \oplus \underline{C}_{*}\left(z_{\text {equi }}\left(X \times V_{2}, r+n\right)\right) \rightarrow\right. \\
\underline{C}_{*}\left(z_{\text {equi }}\left(X \times V_{3}, r+n\right)\right) \rightarrow \underline{C}_{*}\left(z_{\text {equi }}(X \times V, r+n)\right)[1]
\end{gathered}
$$

at $\operatorname{Spec}(k)$. Hence, in the sense of [2], the presheaf $\underline{C}_{*}\left(z_{\text {equi }}(X, r)\right)$ is "pseudoflasque". Thus, we may apply $[2$, Th. 4$]$ to conclude that

$$
\mathbf{H}^{-i}\left(U, \underline{C}_{*}\left(z_{\text {equi }}(X, r)\right)_{Z a r}\right)=h_{i}\left(\underline{C}_{*}\left(z_{\text {equi }}(X, r)\right)(U)\right) .
$$

On the other hand, Theorem 5.5 asserts that the left hand side of the above equality equals $A_{r, i}(U, X)$ whereas the right hand side equals $\underline{h}_{i}\left(z_{\text {equi }}(X, r)\right)(U)$ by definition.

The following theorem provides our strongest duality theorem, in which both $Y$ and $X$ are permited to be arbitrary schemes of finite type over $k$.

Theorem 8.2 Let $k$ be a field which admits resolution of singularities, let $X, Y$ be schemes of finite type over $k$, and let $U$ be a smooth scheme of pure dimension $n$ over $k$. There are canonical isomorphisms

$$
A_{r, i}(Y \times U, X) \rightarrow A_{r+n, i}(Y, X \times U) .
$$


Proof: Let $\mathcal{U}=\left\{U_{i} \rightarrow U\right\}$ be a finite open covering of $U$ by quasi-projective schemes. The complexes $\underline{C}_{*}\left(z\left(U_{i}, X, r\right)\right)$ form a bicomplex and we denote by $\underline{C}_{*}(z(\mathcal{U}, X, r))$ its total complex. It follows immediately from definition of bivariant cycle cohomology groups that there are canonical homomorphisms

$$
\mathbf{H}_{c d h}^{-i}\left(Y, \underline{C}_{*}(z(\mathcal{U}, X, r))_{c d h}\right) \rightarrow A_{r, i}(Y \times U, X) .
$$

On the other hand the duality embeddings $z\left(U_{i}, X, r\right) \rightarrow z\left(X \times U_{i}, r+\right.$ $\operatorname{dim}(U))$ give us together with Theorem 7.4 a canonical quasi-isomorphism of complexes

$$
\underline{C}_{*}(z(\mathcal{U}, X, r))_{c d h} \rightarrow \underline{C}_{*}(z(X \times U, r+\operatorname{dim}(U))) .
$$

It is sufficient therefore to show that the homomorphisms

$$
\mathbf{H}_{c d h}^{-i}\left(Y, \underline{C}_{*}(z(\mathcal{U}, X, r))_{c d h}\right) \rightarrow A_{r, i}(Y \times U, X)
$$

are isomorphisms. Since these homomorphisms are canonical and both sides are cohomology groups in cdh-topology the problem is cdh-local. In particular since $k$ admits resolution of singularities we may assume that $Y$ is smooth and quasi-projective. In this case our statement follows easily from Theorem 8.1 and Theorem 7.4.

We provide additional good properties of the bivariant theory $A_{*, *}(-,-)$ in the next theorem.

Theorem 8.3 Let $k$ be a field which admits resolution of singularities and let $X, Y$ be schemes of finite type over $k$.

1. (Homotopy invariance) The pull-back homomorphism $z_{\text {equi }}(X, r) \rightarrow$ $z_{\text {equi }}\left(X \times \mathbf{A}^{1}, r+1\right)$ induces for any $i \in \mathbf{Z}$ an isomorphism

$$
A_{r, i}(Y, X) \rightarrow A_{r+1, i}\left(Y, X \times \mathbf{A}^{1}\right) .
$$

2. (Suspension) Let

$$
\begin{aligned}
& p: X \times \mathbf{P}^{1} \rightarrow X \\
& i: X \rightarrow X \times \mathbf{P}^{1}
\end{aligned}
$$

be the natural projection and closed embedding. Then the morphism

$$
i_{*} \oplus p^{*}: z_{\text {equi }}(X, r+1) \oplus z_{\text {equi }}(X, r) \rightarrow z_{\text {equi }}\left(X \times \mathbf{P}^{1}, r+1\right)
$$


induces an isomorphism

$$
A_{r+1, i}(Y, X) \oplus A_{r, i}(Y, X) \rightarrow A_{r+1, i}\left(Y, X \times \mathbf{P}^{1}\right)
$$

3. (Cosuspension) There are canonical isomorphisms:

$$
A_{r, i}\left(Y \times \mathbf{P}^{1}, X\right) \rightarrow A_{r+1, i}(Y, X) \oplus A_{r, i}(Y, X) .
$$

4. (Gysin) Let $Z \subset U$ be a closed immersion of smooth schemes everywhere of codimension $c$ in $U$. Then there is a canonical long exact sequence of abelian groups of the form

$$
\begin{gathered}
\ldots \rightarrow A_{r+c, i}(Z, X) \rightarrow A_{r, i}(U, X) \rightarrow A_{r, i}(U-Z, X) \\
\rightarrow A_{r+c, i-1}(Z, X) \rightarrow \ldots
\end{gathered}
$$

\section{Proof:}

1. Since we have natural homomorphisms

$$
A_{r, i}(Y, X) \rightarrow A_{r+1, i}\left(Y, X \times \mathbf{A}^{1}\right),
$$

Propositions 4.4, 4.5 and resolution of singularities imply that we may assume $Y$ to be smooth and quasi-projective. Then (1) follows from Theorems 8.1, 7.4 and Lemma 4.1.

2. This follows from localization exact sequence (5.12) and (1).

3. This follows immediately from Theorem 8.2 and (2).

4. This follows immediately from Theorem 8.2 and Corollary 5.12.

Remark 8.4 The "cosuspension" and "suspension" properties of the bivariant theory $A_{*, *}(-,-)$ are particular cases of projective bundle theorems for the first and second variables respectively. For the corresponding general results see [24]. 
Let $X, X^{\prime}$ be schemes of finite type over $k$ and let $U$ be a smooth scheme over $k$. For any pair of integral closed subschemes $Z \subset X \times U, Z^{\prime} \subset X^{\prime} \times U$ equidimensional over $U$, the fibre product $Z \times_{U} Z^{\prime}$ is also equidimensional over $U$. Thus, sending $Z, Z^{\prime}$ to the cycle associated to the subscheme $Z \times_{U} Z^{\prime} \subset$ $X \times X^{\prime} \times U$ determines a pairing

$$
\times: Z(X, r) \otimes z_{\text {equi }}\left(X^{\prime}, r^{\prime}\right) \rightarrow Z\left(X \times X^{\prime}, r+r^{\prime}\right)
$$

of presheaves.

Using this product pairing, we define the algebraic analogue of the operations introduced in [11] for Lawson homology, formulated in terms of the product pairing in [6], and extended to a bivariant context in [8]. We employ the standard notation $\stackrel{L}{\otimes}$ to denote the derived tensor product.

Proposition 8.5 Let $k$ be a field which admits resolution of singularities and let $X, Y$ be schemes of finite type over $k$. Then there is a natural pairing for any $i \geq 0$

$$
A_{r+1, i}(Y, X) \stackrel{L}{\otimes} A_{0, j}\left(\operatorname{Spec}(k), \mathbf{P}^{1}\right) \rightarrow A_{r, i+j}(Y, X) .
$$

Proof: Our pairing factors through the natural map

$$
A_{r+1, i}(Y, X) \stackrel{L}{\otimes} A_{0, j}\left(\operatorname{Spec}(k), \mathbf{P}^{1}\right) \stackrel{1 \otimes p^{*}}{\rightarrow} A_{r+1, i}(Y, X) \stackrel{L}{\otimes} A_{0, j}\left(Y, \mathbf{P}^{1}\right)
$$

and the projection

$$
A_{r+1, i+j}\left(Y, X \times \mathbf{P}^{1}\right) \rightarrow A_{r, i+j}(Y, X)
$$

associated to the suspension isomorphism of Theorem8.3(2). Thus, it suffices to exhibit a natural pairing

$$
A_{r+1, i}(Y, X) \stackrel{L}{\otimes} A_{0, j}\left(Y, \mathbf{P}^{1}\right) \rightarrow A_{r+1, i+j}\left(Y, X \times \mathbf{P}^{1}\right) .
$$

This pairing is induced by the product pairing

$$
\times: z_{\text {equi }}(X, r+1) \times z_{\text {equi }}\left(\mathbf{P}^{1}, 0\right) \rightarrow z_{\text {equi }}\left(X \times \mathbf{P}^{1}, r+1\right) .
$$

We next use duality to provide

$$
A_{r, i}(X)=A_{r, i}(\operatorname{Spec}(k), X)=\underline{h}_{i}\left(z_{\text {equi }}(x, r)\right)(\operatorname{Spec}(k))
$$


with a natural multiplicative pairing for smooth schemes $X$. This multiplicative structure is inspired by the multiplicative structure on "morphic cohomology" studied in [8].

Proposition 8.6 Let $k$ be a field which admits resolution of singularities and let $X$ be a smooth scheme of pure dimension $n$ over $k$. Then there is a natural multiplication

$$
A_{r, i}(X) \stackrel{L}{\otimes} A_{s, j}(X) \rightarrow A_{r+s-n, i+j}(X) \quad, \quad \text { for any } r+s \geq n .
$$

Proof: By Theorems 8.3(1), 7.4, we have natural isomorphisms

$$
A_{r, i}(\operatorname{Spec}(k), X) \simeq A_{r+n, i}\left(\operatorname{Spec}(k), X \times \mathbf{A}^{n}\right) \simeq A_{r, i}\left(X, \mathbf{A}^{n}\right) .
$$

On the other hand, the product pairing

$$
\times: z_{\text {equi }}\left(\mathbf{A}^{n}, r\right) \otimes z_{\text {equi }}\left(\mathbf{A}^{n}, s\right) \rightarrow z_{\text {equi }}\left(\mathbf{A}^{2 n}, r+s\right)
$$

determines a pairing

$$
A_{r, i}\left(X, \mathbf{A}^{n}\right) \stackrel{L}{\otimes} A_{s, j}\left(X, \mathbf{A}^{n}\right) \rightarrow A_{r+s, i+j}\left(X, \mathbf{A}^{2 n}\right) .
$$

Using Theorems 8.3(1), 7.4 once again, we have

$$
A_{r+s, i+j}\left(X, \mathbf{A}^{2 n}\right) \simeq A_{r+s+n, i+j}\left(X \times \mathbf{A}^{2 n}\right) \simeq A_{r+s-n, i+j}(X) .
$$

Our multiplicative pairing now follows.

We introduce one final pairing on our bivariant cycle cohomology groups. The following "composition pairing" is based on a pairing introduced in [8] and further examined in [3].

Proposition 8.7 Let $k$ be a field which admits resolution of singularities, let $T$ be a scheme of finite type over $k$, and let $U$ be a smooth scheme, and let $X$ be a projective, smooth scheme. Then there is a natural composition pairing of chain complexes

$$
\underline{C}_{*}\left(z_{\text {equi }}(X, r)\right)(U) \otimes \underline{C}_{*}\left(z_{\text {equi }}(T, s)\right)(X) \rightarrow \underline{C}_{*}\left(z_{\text {equi }}(T, r+s)\right)(U)
$$

which induces the pairing

$$
A_{r, i}(U, X) \stackrel{L}{\otimes} A_{s, j}(X, T) \rightarrow A_{r+s, i+j}(U, T) .
$$


Proof: Given by sending a pair of closed integral subschemes

$$
Z \subset X \times U \times \Delta^{n}, W \subset T \times X \times \Delta^{n},
$$

we consider the subscheme

$$
W \times_{X \times \Delta^{n}} Z \subset T \times X \times U \times \Delta^{n} .
$$

We easily verify that if $Z$ is equidimensional over $U \times \Delta^{n}$ and if $W$ is equidimensional over $X \times \Delta^{n}$, then $W \times_{X \times \Delta^{n}} Z$ is equidimensional over $U \times \Delta^{n}$. We define the asserted pairing of chain complexes by sending $(Z, W)$ to the proper push-forward via the projection $T \times X \times U \times \Delta^{n} \rightarrow T \times U \times \Delta^{n}$ of the cycle associated to $W \times_{X \times \Delta^{n}} Z$.

\section{Motivic cohomology and homology.}

In this final section, we introduce four theories defined for schemes of finite type over a field $k$. They are called correspondingly - motivic homology, motivic cohomology, Borel-Moore motivic homology and motivic cohomology with compact supports. All of them but motivic homology (with compact supports) are closely related to bivariant cycle cohomology.

Since some of the definitions below are rather involved, we want to explain first what motivates them. Let $X$ be a scheme of finite type over $k$. As shown in [24] one can construct a certain triangulated category $D M_{g m}(k)$ of "geometrical mixed motives over $k$ " and asociated to any such $X$ two objects in this category: $M(X)$ - the "motive" of $X$ and $M_{c}(X)$ - the "motive with compact supports" of $X$. The correspondence $X \mapsto M(X)$ is covariantly functorial with respect to all morphisms while the correspondence $X \mapsto M_{c}(X)$ is covariantly functorial with respect to proper morphisms and contravariantly functorial with respect to equidimensional morphisms of relative dimension zero. For a proper $X$ one has $M_{c}(X)=M(X)$.

The category $D M_{g m}(k)$ has a tensor structure and a distinguished invertible object $\mathbf{Z}(1)$ called the Tate object. For any object $M$ in $D M_{g m}(k)$ we denote by $M(n)$ the object $M \otimes \mathbf{Z}(1)^{\otimes n}$. In particular $\mathbf{Z}(n)=\mathbf{Z}(1)^{\otimes n}$.

Using this formalism, one could define various motivic theories as follows:

\section{Motivic cohomology:}

$$
H^{j}(X, \mathbf{Z}(i))=H_{D M}(M(X), \mathbf{Z}(i)[j])
$$




\section{Motivic cohomology with compact supports:}

$$
H_{c}^{j}(X, \mathbf{Z}(i))=\operatorname{Hom}_{D M}\left(M_{c}(X), \mathbf{Z}(i)[j]\right)
$$

Motivic homology:

$$
H_{j}(X, \mathbf{Z}(i))=\operatorname{Hom}_{D M}(\mathbf{Z}(i)[j], M(X))
$$

\section{Borel-Moore motivic homology:}

$$
H_{j}^{B M}(X, \mathbf{Z}(i))=\operatorname{Hom}_{D M}\left(\mathbf{Z}(i)[j], M_{c}(X)\right)
$$

The definitions given below are just "explicit" reformulations of these heuristic ones. There are two main reasons why they are more involved than one might expect. The first which applies mainly to the case of homology is that the object $\mathbf{Z}(i)$ has no geometrical meaning for $i<0$ and $\operatorname{Hom}(\mathbf{Z}(i)[j], M(X))$ is in this case just a formal notation for $\operatorname{Hom}(\mathbf{Z}[j], M(X)(-i))$. Thus we have to distinguish the cases $i<0$ and $i \geq 0$ in the definitions below. The second is that there are several ways to interpret $\mathbf{Z}(i)$ geometrically for $i>0$. In Definitions 9.2, 9.3 below, we use the fact that $\mathbf{Z}(i)=M_{c}\left(\mathbf{A}^{i}\right)[-2 i]$ and in Definition 9.4 the fact that $M\left(\mathbf{A}^{i}-\{0\}\right)=\mathbf{Z}(i)[2 i-1] \oplus \mathbf{Z}$.

Definition 9.1 Let $X$ be a scheme of finite type over $k$. For any $n, r \in \mathbf{Z}$ we define the Borel-Moore motivic homology of $X$ as

$$
H_{n}^{B M}(X, \mathbf{Z}(r)) \equiv\left\{\begin{array}{cl}
A_{r, n-2 r}(X) & \text { for } r \geq 0 \\
A_{0, n-2 r}\left(X \times \mathbf{A}^{-r}\right) & \text { for } r \leq 0 .
\end{array}\right.
$$

Definition 9.2 Let $X$ be a scheme of finite type over $k$. For all $m, s \in \mathbf{Z}$, we define the motivic cohomology of $X$ as

$$
H^{m}(X, \mathbf{Z}(s)) \equiv A_{0,2 s-m}\left(X, \mathbf{A}^{s}\right)
$$

$\left(\right.$ for $s<0$ we set $\left.A_{0,2 s-m}\left(X, \mathbf{A}^{s}\right)=0\right)$.

To define motivic cohomology with compact supports we use the construction of cdh-cohomology with compact supports given at the end of Section 3 . 
Definition 9.3 Let $X$ be a scheme of finite type over $k$. For any $m, s \in \mathbf{Z}$ we define the motivic cohomology of $X$ with compact supports of $X$ as

$$
H_{c}^{m}(X, \mathbf{Z}(s)) \equiv \mathbf{H}_{c}^{m-2 s}\left(X, \underline{C}_{*}\left(z_{\text {equi }}\left(\mathbf{A}^{s}, 0\right)\right)\right) .
$$

To define the last of our four theories, namely motivic homology (with compact supports) we need to remind first the following definition of algebraic singular homology (see [22]).

For any scheme of finite type $X$ over $k$, denote by $c_{\text {equi }}(X, 0)$ the subpresheaf in $z_{\text {equi }}(X, 0)$ whose values on a smooth scheme $U$ over $k$ the subgroup $c_{\text {equi }}(X, 0)(U)$ is generated by integral closed subschemes $Z$ in $U \times X$ which are proper over $U$ (note that this implies that $Z$ is in fact finite over $U$ ). One can easily see that unlike $z_{\text {equi }}(X, 0)$ the presheaf $c_{\text {equi }}(X, 0)$ is covariantly functorial with respect to all morphisms $X_{1} \rightarrow X_{2}$ and contravariantly functorial only with respect to flat proper morphisms. For any $X$ the presheaf $c_{\text {equi }}(X, 0)$ has a structure of a pretheory obtained by restricting of the canonical structure of a pretheory on $z_{\text {equi }}(X, 0)$. Note also that if $X$ is proper we have $c_{\text {equi }}(X, 0)=z_{\text {equi }}(X, 0)$. We define the algebraic singular complex of $X$ as the complex of sheaves $\underline{C}_{*}\left(c_{\text {equi }}(X, 0)\right.$ ). Its homology sheaves (or more precisely their sections on $\operatorname{Spec}(k)$ ) are algebraic singular homology of $X$ (cf. $[22])$. They form a homology theory which is a part of our motivic homology of $X$.

Generalizing algebraic singular homolgy we have:

Definition 9.4 Let $X$ be a scheme of finite type over $k$. For any $n, r \in \mathbf{Z}$ we define motivic homology of $X$ as follows:

$$
H_{n}(X, \mathbf{Z}(r)) \equiv \begin{cases}\mathbf{H}_{\{0\}}^{2 r-n}\left(\mathbf{A}^{r}, \underline{C}_{*}\left(c_{\text {equi }}(X, 0)\right)\right) & r \geq 0 \\ h_{n-2 r-1}\left(\underline{C}_{*}\left(c_{\text {equi }}\left(X \times\left(\mathbf{A}^{r}-\{0\}\right), 0\right) / c_{\text {equi }}(X \times\{1\}, 0)\right)\right) & r \leq 0\end{cases}
$$

In what follows, we summarize some basic properties of our four theories in the case when $k$ admits resolution of singularities. All of these properties follow from results of this paper, [23] and the corresponding properties of the motivic category considered in [24].

Motivic cohomology - is a family of contravariant functors $H^{i}(-, \mathbf{Z}(j))$ from the category $S c h / k$ to the category of abelian groups. These fuctors have the following basic properties: 
Homotopy invariance. For any scheme $X$ of finite type over $k$ one has canonical isomorphisms $H^{i}\left(X \times \mathbf{A}^{1}, \mathbf{Z}(j)\right)=H^{i}(X, \mathbf{Z}(j))$.

Algebraic suspension property. For any $X$ as above, one has canonical isomorphisms

$$
H^{i}\left(X \times \mathbf{P}^{1}, \mathbf{Z}(j)\right)=H^{i}(X, \mathbf{Z}(j)) \oplus H^{i-2}(X, \mathbf{Z}(j-1)) .
$$

Mayer-Vietoris exact sequence. For any $X$ as above and any open covering $X=U \cup V$ of $X$, there is a canonical long exact sequence of the form:

$$
\begin{aligned}
\ldots \rightarrow H^{i}(X, \mathbf{Z}(j)) \rightarrow & H^{i}(U, \mathbf{Z}(j)) \oplus H^{i}(V, \mathbf{Z}(j)) \rightarrow H^{i}(U \cap V, \mathbf{Z}(j)) \\
& \rightarrow H^{i+1}(X, \mathbf{Z}(j)) \rightarrow \ldots
\end{aligned}
$$

Blow-up exact sequence. For any schemes $X, Y$ of finite type over $k$, proper morphism $p: X \rightarrow Y$ and closed subscheme $Z \rightarrow Y$ in $Y$ such that $p^{-1}(Y-Z) \rightarrow Y-Z$ is an isomorphism, there is a long exact sequence of the form:

$$
\begin{gathered}
\ldots \rightarrow H^{i}(Y, \mathbf{Z}(j)) \rightarrow H^{i}(X, \mathbf{Z}(j)) \oplus H^{i}(Z, \mathbf{Z}(j)) \rightarrow \\
H^{i}\left(p^{-1}(Z), \mathbf{Z}(j)\right) \rightarrow H^{i+1}(Y, \mathbf{Z}(j)) \rightarrow \ldots
\end{gathered}
$$

Covariant functoriality. For a proper, flat, equidimensional morphism $f: X \rightarrow Y$ of relative dimension $d$, there are homomorphisms

$$
H^{i}(X, \mathbf{Z}(j)) \rightarrow H^{i-2 d}(X, \mathbf{Z}(j-d)) .
$$

Motivic homology - is a family of covariant functors $H_{i}(-, \mathbf{Z}(j))$ from the category $S c h / k$ to the category of abelian groups. These fuctors have the following basic properties:

Homotopy invariance. For any scheme $X$ of finite type over $k$ one has canonical isomorphisms $H_{i}\left(X \times \mathbf{A}^{1}, \mathbf{Z}(j)\right)=H_{i}(X, \mathbf{Z}(j))$.

Algebraic suspension property. For any $X$ as above, one has canonical isomorphisms

$$
H_{i}\left(X \times \mathbf{P}^{1}, \mathbf{Z}(j)\right)=H_{i}(X, \mathbf{Z}(j)) \oplus H_{i-2}(X, \mathbf{Z}(j-1)) .
$$


Mayer-Vietoris exact sequence. For any $X$ as above and any open covering $X=U \cup V$ of $X$, there is a canonical long exact sequence of the form:

$$
\begin{aligned}
\ldots \rightarrow H_{i}(U \cap V, \mathbf{Z}(j)) & \rightarrow H_{i}(U, \mathbf{Z}(j)) \oplus H_{i}(V, \mathbf{Z}(j)) \rightarrow H_{i}(X, \mathbf{Z}(j)) \\
\rightarrow & H^{i-1}(U \cap V, \mathbf{Z}(j)) \rightarrow \ldots
\end{aligned}
$$

Blow-up exact sequence. For any schemes $X, Y$ of finite type over $k$, a proper morphism $p: X \rightarrow Y$ and a closed subscheme $Z \rightarrow Y$ in $Y$ such that $p^{-1}(Y-Z) \rightarrow Y-Z$ is an isomorphism there is a long exact sequence of the form:

$$
\begin{gathered}
\ldots \rightarrow H_{i}\left(p^{-1}(Z), \mathbf{Z}(j)\right) \rightarrow H_{i}(X, \mathbf{Z}(j)) \oplus H_{i}(Z, \mathbf{Z}(j)) \rightarrow \\
H^{i}(Y, \mathbf{Z}(j)) \rightarrow H^{i-1}\left(p^{-1}(Z), \mathbf{Z}(j)\right) \rightarrow \ldots
\end{gathered}
$$

Contravariant functoriality. For a proper flat equidimensional morphism $f: X \rightarrow Y$ of relative dimension $d$, there are homomorphisms

$$
H_{i}(Y, \mathbf{Z}(j)) \rightarrow H_{i+2 d}(X, \mathbf{Z}(j+d)) .
$$

Motivic cohomology with compact support - is a family of contravariant functors $H_{c}^{i}(-, \mathbf{Z}(j))$ from the category $S c h_{c} / k$ of schemes and proper morphisms to the category of abelian groups. These fuctors have the following basic properties:

Homotopy invariance. For any scheme of finite type over $k$, one has canonical isomorphisms $H_{c}^{i}\left(X \times \mathbf{A}^{1}, \mathbf{Z}(j)\right)=H_{c}^{i-2}(X, \mathbf{Z}(j-1))$.

Localization. For any $X$ as above and any closed subscheme $Z$ in $X$, one has a canonical exact sequence:

$$
\begin{gathered}
\ldots \rightarrow H_{c}^{i}(X-Z, \mathbf{Z}(j)) \rightarrow H_{c}^{i}(X, \mathbf{Z}(j)) \rightarrow H_{c}^{i}(Z, \mathbf{Z}(j)) \\
\rightarrow H_{c}^{i+1}(Z, \mathbf{Z}(j)) \rightarrow \ldots
\end{gathered}
$$

Covariant functoriality. For a flat equidimensional morphism $f: X \rightarrow$ $Y$ of relative dimension $d$, there are homomorphisms

$$
H_{c}^{i}(X, \mathbf{Z}(j)) \rightarrow H_{c}^{i-2 d}(X, \mathbf{Z}(j-d)) .
$$


Borel-Moore motivic homology - is a family of covariant functors $H_{i}^{B M}(-, \mathbf{Z}(j))$ from the category $S c h_{c} / k$ to the category of abelian groups. These fuctors have the following basic properties:

Homotopy invariance. For any scheme of finite type over $k$, one has canonical isomorphisms $H_{i}^{B M}\left(X \times \mathbf{A}^{1}, \mathbf{Z}(j)\right)=H_{i-2}^{B M}(X, \mathbf{Z}(j))$.

Localization. For any $X$ as above and any closed subscheme $Z$ in $X$, one has a canonical exact sequence:

$$
\begin{aligned}
\ldots \rightarrow H_{i}^{B M}(Z, \mathbf{Z}(j)) & \rightarrow H_{i}^{B M}(X, \mathbf{Z}(j)) \rightarrow H_{i}^{B M}(X-Z, \mathbf{Z}(j)) \\
\rightarrow & H_{i+1}^{B M}(Z, \mathbf{Z}(j)) \rightarrow \ldots
\end{aligned}
$$

Contravariant functoriality. For a flat equidimensional morphism

$f: X \rightarrow Y$ of relative dimension $d$, there are homomorphisms

$$
H_{i}^{B M} c(Y, \mathbf{Z}(j)) \rightarrow H_{i+2 d}^{B M}(X, \mathbf{Z}(j+d)) .
$$

The following two main results relate our four theories.

1. If $X$ is a proper scheme of finite type over $k$, then one has canonical isomorphisms

$$
\begin{gathered}
H_{c}^{i}(X, \mathbf{Z}(j))=H^{i}(X, \mathbf{Z}(j)) \\
H_{i}^{B M}(X, \mathbf{Z}(j))=H_{i}(X, \mathbf{Z}(j))
\end{gathered}
$$

2. If $X$ is a smooth scheme of finite type over $k$ of pure dimension $d$ and $Z$ is a closed subscheme in $X$, then there are long exact sequences:

$$
\begin{gathered}
\ldots \rightarrow H_{i}(X-Z, \mathbf{Z}(j)) \rightarrow H_{i}(X, \mathbf{Z}(j)) \rightarrow H_{c}^{2 d-i}(Z, \mathbf{Z}(d-j)) \\
\rightarrow H_{i-1}(X-Z, \mathbf{Z}(j)) \rightarrow \ldots \\
\ldots \rightarrow H_{2 d-i}^{B M}(Z, \mathbf{Z}(d-j)) \rightarrow H^{i}(X, \mathbf{Z}(j)) \rightarrow H^{i}(X-Z, \mathbf{Z}(j)) \\
\rightarrow H_{2 d-i-1}^{B M}(Z, \mathbf{Z}(d-j)) \rightarrow \ldots
\end{gathered}
$$

In particular if $X$ is a smooth scheme of finite type over $k$ one has canonical isomorphisms

$$
\begin{aligned}
& H_{i}(X, \mathbf{Z}(j))=H_{c}^{2 d-i}(Z, \mathbf{Z}(d-j)) \\
& H^{i}(X, \mathbf{Z}(j))=H_{2 d-i}^{B M}(Z, \mathbf{Z}(d-j)) .
\end{aligned}
$$


Remark 9.5 One can also define motivic (co-)homology theories for coefficients other than $\mathbf{Z}$. More precisely, for any commutative ring $R$ one can define theories $H^{i}(X, R(j)), H_{i}(X, R(j))$ etc. in such a way that the usual universal coefficients theorems hold.

\section{References}

[1] S. Bloch. Algebraic cycles and higher K-theory. Adv. in Math., 61:267304, 1986.

[2] K.S. Brown and S.M. Gersten. Algebraic K-theory and generalizied sheaf cohomology. Lecture Notes in Math. 341, pages 266-292, 1973.

[3] E. Friedlander. Filtrations on algebraic cycles and homology. To appear in Annales des Ecole Norm. Sup.

[4] E. Friedlander. Algebraic cycles, Chow varieties, and Lawson homology. Compositio Math., 77:55-93, 1991.

[5] E. Friedlander. Some computations of algebraic cycle homology. Ktheory, 8:271-285, 1994.

[6] E. Friedlander and O. Gabber. Cycle spaces and intersection theory. Topological methods in modern mathematics, pages 325-370, 1993.

[7] E. Friedlander and H. B. Lawson. Duality for spaces of algebraic cycles. Preprint.

[8] E. Friedlander and H. B. Lawson. A theory of algebraic cocycles. Annals of Math., 136:361-428, 1992.

[9] E. Friedlander and H. B. Lawson. A moving lemma for families of algebraic cycles. Preprint, 1994.

[10] E. Friedlander and B. Mazur. Correspondence homomorphisms for singular varieties. Annales d'Institut Fourier, 44:703-727, 1994.

[11] E. M. Friedlander and B. Mazur. Filtrations on the homology of algebraic varieties, volume 529 of Memoir of the AMS. AMS, Providence, RI, 1994. 
[12] H. Hironaka. Resolution of singularities of an algebraic variety over a field of characteristic zero. Ann. of Math., 79:109-326, 1964.

[13] H.B. Lawson. Algebraic cycles and homotopy theory. Ann. of Math., 129:599-632, 1989.

[14] M. Nagata. Imbedding of an abstract variety in a complete variety. $J$. Math. Kyoto Univ., 2:1-10, 1962.

[15] E. Nart. The Bloch complex in codimension one and arithmetic duality. Journal of Number Theory, 32(3):321-331, 1989.

[16] Y. Nisnevich. The completely decomposed topology on schemes and associated descent spectral sequences in algebraic K-theory. In Algebraic K-theory: connections with geometry and topology, pages 241-342. Kluwer Acad. Publ., Dordrecht, 1989.

[17] M. Raynaud and L. Gruson. Criteres de platitude et de projectivite. Inv. Math., 13:1-89, 1971.

[18] J. Roberts. Chow's moving lemma. In Algebraic geometry, pages 89-96. Oslo, 1970.

[19] P. Samuel. Methodes d'algebre abstraite en geometrie algebrique. Ergebnisse der Mathematik, N.F.4. Springer-Verlag, Berlin, 1955.

[20] A. Suslin. Higher Chow groups and etale cohomology. This volume, page ...

[21] A. Suslin and V. Voevodsky. Relative cycles and Chow sheaves. This volume, page ...

[22] A. Suslin and V. Voevodsky. Singular homology of abstract algebraic varieties. Preprint., 1992.

[23] V. Voevodsky. Cohomological theory of presheaves with transfers. This volume, page ...

[24] V. Voevodsky. Triangulated categories of motives over a field. This volume, page ...

[25] V. Voevodsky. Homology of schemes I. Preprint., 1993. 